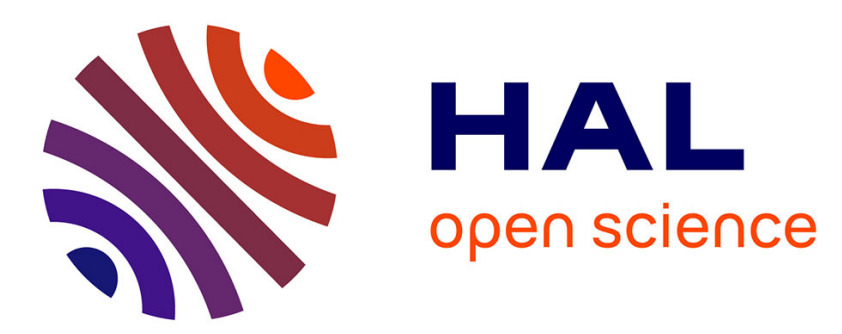

\title{
Recherche multi-acteurs et transdisciplinaire pour des systèmes alimentaires bio et locaux
}

Véronique Chable, Estelle Serpolay

\section{To cite this version:}

Véronique Chable, Estelle Serpolay. Recherche multi-acteurs et transdisciplinaire pour des systèmes alimentaires bio et locaux. Techniques de l'Ingénieur. Eco-Conception et Innovation Responsable, 2016. hal-02631698

\section{HAL Id: hal-02631698 \\ https://hal.inrae.fr/hal-02631698}

Submitted on 27 May 2020

HAL is a multi-disciplinary open access archive for the deposit and dissemination of scientific research documents, whether they are published or not. The documents may come from teaching and research institutions in France or abroad, or from public or private research centers.
L'archive ouverte pluridisciplinaire HAL, est destinée au dépôt et à la diffusion de documents scientifiques de niveau recherche, publiés ou non, émanant des établissements d'enseignement et de recherche français ou étrangers, des laboratoires publics ou privés. 


\title{
(i)
}

\section{TECHNIQUES}

\section{DE L'INGENIEUR}

\author{
Réf.: AG103 V1
}

Date de publication

10 janvier 2016

\section{Recherche multi-acteurs et transdisciplinaire pour des systèmes alimentaires bio et locaux}

\author{
Cet article est issu de : Innovation I Éco-conception et innovation responsable
}

\author{
par Véronique CHABLE, Estelle SERPOLAY
}

\author{
Mots-clés \\ Biodiversité cultivée | \\ recherche participative | \\ multi-acteurs
}

\section{Keywords}

Cultivated biodiversity | participatory research | multi-actor

\begin{abstract}
Résumé L'agriculture biologique connaît un fort développement mais peu de recherches lui sont dédiées en France. La notion d'agriculture biologique regroupe des conceptions très diverses dans un contexte d'agriculture conventionnelle dominante et d'une politique de « transition agroécologique ». La biodiversité cultivée, réduite par l'intensification de l'agriculture, est la clé de la performance des agrosystèmes « bio » basés sur le respect et la maximisation des processus vitaux. Pour pallier le manque de variétés adaptées et diversifiées, une collaboration avec les paysans « bio » a été initiée. Cet article présente les expériences et les évolutions du métier d'«ingénieur du vivant » élargi aux systèmes alimentaires locaux et leurs acteurs, avec une approche transdisciplinaire et multi-acteurs.
\end{abstract}

Pour toute question : Service Relation clientèle Techniques de l'Ingénieur Immeuble Pleyad 1 39. boulevard Ornano 93288 Saint-Denis Cedex

Par mail :

infos.clientsateching.com Par téléphone :

0033 (0)153352020
Document téléchargé le : 15/03/2017

Pour le compte : 7200035215 - agrocampus // nelson DAURELLE // 147.99.187.242 


\title{
Recherche multi-acteurs et transdisciplinaire pour des systèmes alimentaires bio et locaux
}

\author{
par Véronique CHABLE \\ Ingénieur de recherche à I'INRA (Institut national de la recherche agronomique) \\ Unité de recherche INRA SAD paysage, équipe Biodiversité cultivée et recherche \\ participative INRA-ITAB, Rennes, France \\ et Estelle SERPOLAY \\ Chargée de mission à I'ITAB (Institut technique de I'agriculture biologique) \\ Unité de recherche INRA SAD paysage, équipe Biodiversité cultivée et recherche \\ participative INRA-ITAB, Rennes, France
}

\begin{tabular}{|c|c|c|}
\hline 1. & Agriculture biologique : conceptions plurielles ........................ & AG $103-2$ \\
\hline 2. & Pour une recherche spécifiquement dédiée à l'AB....................... & - \\
\hline 3. & $\begin{array}{l}\text { Hypothèse : la diversité comme fondement } \\
\text { d'une agriculture vivante }\end{array}$ & - \\
\hline 4. & $\begin{array}{l}\text { Notre métier : la diversité des savoirs au cœur du processus } \\
\text { de recherche }\end{array}$ & - \\
\hline 5. & Conclusion .............................. & - \\
\hline 6. & 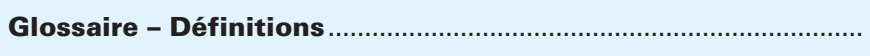 & - \\
\hline Pol & en savoir plus. & Doc. AG 103 \\
\hline
\end{tabular}

epuis les années 1990, l'agriculture biologique (AB) ne montre que des chif-
fres en progression que ce soit en termes de surface, du nombre de fermes
ou de consommateurs. Dans un contexte environnemental perturbé - réduction
de la biodiversité, pollutions et changement climatique - couplé à des question-
nements croissants sur la qualité de l'alimentation, l'AB offre une alternative
rassurante aux citoyens inquiets pour l'avenir de la planète et de leur santé. Mais
qu'est-ce que l'agriculture biologique? Quels sont ses principes, ses différentes
formes? Répondent-elles aux questions de société actuelles? Quels moyens
sont mis en oeuvre pour son développement et sur quelles hypothèses? Le succès d'aujourd'hui est le fruit de ceux qui la pratiquent. Les efforts de recherche sont restés modestes depuis sa reconnaissance officielle en 1980 en France. Les questions scientifiques qu'elle soulève sont novatrices, car contrairement à ce que certains prétendent, l'agriculture biologique n'est pas celle qui précède l'agriculture conventionnelle, c'est-à-dire avant son intensification. Il ne s'agit pas de refaire l'agriculture d'avant-guerre mais d'utiliser des bases scientifiques, techniques et conceptuelles de différents horizons, de combiner des savoirs et savoir-faire traditionnels et contemporains, pour inventer de nouvelles pratiques qui soient productives, respectueuses de l'environnement et des hommes. L'agriculture biologique est donc un espace de découvertes et d'innovations immense qui nécessite la mobilisation d'une grande diversité de connaissances dans de nombreux domaines. Mais quel est l'état de la recherche pour l'agriculture biologique? Existe-t-il une recherche spécifique pour cette agriculture? Quelles connaissances sont produites, pour qui, pour quoi et pourquoi?

Notre équipe de recherche est spécifiquement dédiée au développement d'une agriculture biologique et paysanne, dont les principes sont hérités des 
pionniers du début du $X X^{e}$ siècle. Elle appelle un regard renouvelé sur la vie et implique une approche adaptée. Notre double compétence, I'une thématique attachée au renouvellement de la biodiversité cultivée et l'autre organisationnelle promouvant une recherche participative, nous a amenées, au fil du temps, des projets et des rencontres, à élargir notre conception de la recherche, passant de l'étude de la diversité des plantes cultivées à l'étude des produits "du champ à l'assiette " et intégrant de plus en plus d'acteurs, avec des savoirs et savoir-faire variés et complémentaires, tout au long du processus de recherche. Notre travail est aujourd'hui devenu une recherche multi-acteurs et transdisciplinaire, basée sur l'action pour des systèmes alimentaires biologiques et locaux, et c'est cette expérience d'ingénieurs du vivant que nous proposons de partager ici.

\section{Agriculture biologique : conceptions plurielles}

Le contexte agricole dans lequel s'insère notre recherche est celui de I'agriculture biologique (AB). Nous utiliserons I'expression complète " agriculture biologique " pour évoquer le concept sous toutes ses formes. Mais plus souvent, nous mentionnerons une désignation simplifiée, "la bio ", très utilisée par ceux qui la pratiquent et la consomment, et font référence à sa forme paysanne et initiale. Faire référence à son histoire est important pour comprendre ses fondements, ses différentes définitions et son positionnement par rapport à l'agriculture conventionnelle, dominante dans nos paysages agricoles européens, et à l'agroécologie, nouveau concept très utilisé dans le contexte scientifique et politique de notre époque.

\subsection{De I'émergence aux cahiers des charges}

En France, le ministère de l'Agriculture a reconnu officiellement I'agriculture biologique en 1980 et il a fallu attendre, au niveau européen, 1992. Cette officialisation s'est traduite par la mise en place de cahiers des charges européens et nationaux. Aux États-Unis, la période 1979-1990 est aussi celle de la reconnaissance officielle [1]. Cette traduction réglementaire a facilité l'essor de l'agriculture biologique mais n'en reflète pas tous ses fondements.

L'agriculture biologique a de multiples facettes que son histoire nous aide à comprendre. L'émergence de la nécessité d'une agriculture respectueuse de l'environnement date de presque un siècle, avec Rudolf Steiner qui créa le concept d'agriculture biodynamique en Allemagne appuyé sur une approche globale des êtres vivants, de la terre et du cosmos. Un autre pionnier marquant fut Sir Albert Howard qui, dans son testament agricole en 1943 [2], proposa aussi de considérer le lien fort entre tous les éléments de I'agroécosystème : "La connaissance de la relation qui existe entre un sol fertile et des cultures saines, des animaux en bonne santé et, enfin et surtout, des êtres humains en bonne santé doit être diffusée partout et largement " ("The connexion which exists between a fertile soil and healthy crops, healthy animals and, last but not least, healthy human beings must be made known far and wide"). Si le lien au sol est fondamental pour ces deux chercheurs, une dimension politique s'est aussi imposée avec les réflexions du Suisse Hans Müller et de sa femme dénonçant les travers socio-économiques et la réduction d'autonomie des agriculteurs suite au développement industriel de l'agriculture; avec Hans Peter Rusch, microbiologiste, ils proposèrent une agriculture organo-biologique basée sur la microbiologie des sols. Ils furent à l'origine du développement de I'agriculture biologique dans les pays germaniques. En
France, les promoteurs historiques de la bio sont Raoul Lemaire et Jean Boucher. Raoul Lemaire a créé la première filière bio au début des années 1930 avec une pratique de fertilisation des sols à base de lithothamne (une algue calcaire riche en magnésium, Lithothamnium calcareum, aussi appelée "maërl ") et en promouvant la sélection et la multiplication de blés à haute valeur boulangère adaptés à une filière bio. A partir de 1964, Raoul Lemaire a fait équipe avec un autre pionnier, Jean Boucher qui avait créé en 1958 le premier Groupement de I'agriculture biologique (GAB) de I'Ouest et en 1961 l'Association française d'agriculture biologique (Afab).

Ainsi, plusieurs mouvements se sont emparés de ces inspirations pour initier des modes de productions biologiques dans les années 1960-1970, bases ensuite de cahiers des charges officiels, élaborés entre 1980 et 1990 en France et en Europe [3]. La nécessité de construction de ces cahiers des charges s'est imposée au fil du temps pour traduire les principes de I'AB en pratiques communes et ordonnées. Les premières règles mises en place étaient issues des pratiques inspirées par les pionniers et la certification était une démarche participative (associative, privée et citoyenne). C'est le cas du label "Demeter " en France en 1979 (mais mentionné dès 1932 à Munich) pour les biodynamistes (issus de la conception anthroposophique de Steiner) et du label "Nature \& Progrès " créé en 1964 en France. Ce sont ces initiatives dans l'esprit des pionniers de la bio qui ont notamment permis l'émergence d'une organisation mondiale des mouvements de I'agriculture biologique (IFOAM - International Federation of Organic Movements). Ces labels historiques et leur organisation spécifique du contrôle sont toujours présents sur le marché et côtoient aujourd'hui le cahier des charges officiel européen (initié en France avec la loi d'orientation agricole de 1980). Le cahier des charges a été créé dans un contexte de développement de I'AB pour apporter une garantie sur les méthodes de travail des agriculteurs aux clients de plus en plus éloignés et distinguer l'agriculture biologique de l'agriculture conventionnelle. Les États sont donc intervenus pour réglementer ce type de production, puis pour harmoniser les pratiques à l'échelle européenne et élargir le marché de l'AB (avec une certification par un tiers et non plus participative). Avec la réglementation des Etats, on observe un changement de logique dans lequel la pratique se met au service d'un marché alors que l'agriculture biologique des pionniers est d'abord une agriculture respectueuse de l'environnement [3]. Certains parlent de la " conventionnalisation de l'agriculture biologique " [4] qui est évoquée pour décrire un secteur $A B$ qui s'éloignerait des valeurs et des pratiques des acteurs historiques pour satisfaire des distributeurs et les opérateurs industriels récemment arrivés sur ce marché, lesquels inciteraient une forme plus conventionnelle de I'agriculture biologique. II est par ailleurs intéressant de noter que le cahier des charges européen de I'AB la résume souvent à une somme d'interdictions (de produits chimiques de synthèse, d'OGM) alors que les labels issus des concepts des pionniers sont ancrés dans une maximisation des processus vitaux. 
Plusieurs cahiers des charges de l'agriculture biologique se côtoient donc aujourd'hui, reflets d'une diversité de conceptions de I'AB qui résultent de son histoire, qui est à lire en regard de celle de l'agriculture en général, et notamment de son évolution industrielle.

\section{Encadré 1 - Diversité des définitions officielles de l'agriculture biologique}

Les définitions officielles/institutionnelles de I'AB sont loin d'en représenter la diversité des formes historiques. Elles sont cependant variées comme le montre ce panel :

Soil Association : "Notre définition de l'agriculture biologique reconnaît les liens directs qui existent entre notre santé et la façon dont notre nourriture est produite. Les fertilisants artificiels sont interdits et les agriculteurs développent un sol fertile par des rotations, l'utilisation de compost, de fumier et de trèfle. Des règles strictes, appelées "standards ", définissent ce que peuvent faire ou ne pas faire les agriculteurs bio - et portent une attention forte à la protection de la faune sauvage et de l'environnement. Tirant son nom de la matière organique (agriculture biologique $=$ organic farming en anglais, NDLR) que les agriculteurs utilisent comme alternative aux produits de synthèse, les agriculteurs bio ont une approche holistique qui, par principe respecte et s'attache au pouvoir des processus naturels pour construire une santé positive à travers l'écologie de la ferme".

IFOAM (International Federation of Organic Agriculture Movements) : " L'agriculture biologique est un système de production qui maintient et améliore la santé des sols, des écosystèmes et des personnes. Elle s'appuie sur des processus écologiques, la biodiversité et des cycles adaptés aux conditions locales, plutôt que sur l'utilisation d'intrants ayant des effets adverses. L'agriculture biologique allie tradition, innovation et science au bénéfice de l'environnement commun et promeut des relations justes et une bonne qualité de vie pour tous ceux qui y sont impliqués ". Cette définition a été élaborée à partir des quatre principes de base de la fédération de l'agriculture bio qui sont la santé, l'écologie, l'équité et la précaution.

FAO (Food and Agriculture Organisation, Nations unies) : "L'agriculture biologique est un système de gestion holistique de la production qui favorise la santé de l'agrosystème, y compris la biodiversité, les cycles biologiques et les activités biologiques des sols. Elle privilégie les pratiques de gestion plutôt que les méthodes de production d'origine extérieure, en tenant compte du fait que les systèmes locaux doivent s'adapter aux conditions régionales. Dans cette optique, des méthodes culturales, biologiques et mécaniques sont, dans la mesure du possible, utilisées de préférence aux produits de synthèse, pour remplir toutes les fonctions spécifiques du système" (Commission du Codex alimentaire FAO/OMS, 1999).

Ministère de I'Agriculture français : "L'agriculture biologique constitue un mode de production qui trouve son originalité dans le recours à des pratiques culturales et d'élevage soucieuses du respect des équilibres naturels. Ainsi, elle exclut l'usage des produits chimiques de synthèse, des OGM et limite l'emploi d'intrants".

Il existe par ailleurs une grande diversité de formes d'agricultures biologiques, qui répondent aux différentes définitions ci-dessus du point de vue des méthodes et des approches, mais ont chacune leur orientation spécifique en fonction du contexte culturel ou philosophique. On peut citer par exemple I'agriculture biodynamique, la permaculture, I'agriculture agroécologique (sous certaines formes), I'agriculture naturelle... Tout cela contribue à la diversité de l'agriculture bio.

\subsection{Agriculture biologique et agriculture conventionnelle}

Dès son origine, l'agriculture biologique est venue en opposition à l'agriculture conventionnelle. Ce sont les conséquences néfastes pour les sols, l'environnement, la santé des plantes, des animaux et des hommes ainsi que les bouleversements sociaux amenés par I'industrialisation de l'agriculture qui ont fait se lever les pionniers dans la première moitié du $\mathrm{XX}^{\mathrm{e}}$ siècle.

Premier paragraphe de la préface du Testament Agricole d'Howard, 1943 [2] :

"Depuis la révolution industrielle, le processus de "croissance" a été intensifié pour produire la nourriture et les matières premières nécessaires à la population et à I'industrie. Rien n'a été fait pour pallier la perte de fertilité des sols concomitante à cette forte augmentation des productions végétale et animale. Les conséquences ont été désastreuses. L'agriculture est devenue déséquilibrée, la terre est "en révolte ": des maladies de toutes sortes apparaissent; dans de nombreux endroits du monde, les sols dégradés disparaissent par l'érosion. Le but de cet ouvrage est d'attirer l'attention sur la destruction du capital de la terre - le sol - de montrer quelques-unes des conséquences du phénomène, et de proposer des méthodes pour restaurer puis entretenir la fertilité perdue."

Premières lignes de la préface du Cours aux agriculteurs de Steiner, $1924[5]$ :

"En 1922-1923, Ernst Stegemann et un groupe d'agriculteurs sont allés demander l'avis de Rudolf Steiner à propos de la "dégénérescence" croissante qu'ils observaient au sein des semences et de nombreuses plantes cultivées. Que faire pour vérifier ce déclin et pour améliorer la qualité des semences et des aliments? Telle fut leur question. Ils ont porté à son attention les faits marquants suivants: les luzernières traditionnellement cultivées pouvaient être exploitées pendant trente ans de suite dans le même champ. Ce délai de trente années est tombé à neuf, puis à sept. Puis arriva l'époque où il était considéré comme un exploit de maintenir cette culture au même endroit pendant quatre ou cinq ans. Aussi, les agriculteurs avaient l'habitude d'ensemencer les cultures annuelles, année après année, à partir de leurs propres semences de seigle, de blé, d'avoine et d'orge. Maintenant, il se trouve qu'ils doivent recourir à de nouvelles "souches " de semences au bout de quelques années." .

Ces deux introductions donnent deux exemples des conséquences perturbatrices de l'industrialisation de l'agriculture : une chute de fertilité des sols, d'une part, et une diminution de la longévité des populations cultivées (de leur vitalité), obligeant les agriculteurs à se réapprovisionner en semences beaucoup plus souvent d'autre part. Elles évoquent le cœur de nos activités de recherche pour le développement de l'agriculture biologique: sélectionner des plantes et développer des pratiques qui favorisent la résilience de l'agroécosystème et des plantes robustes, en y ajoutant la dimension qualité des produits qui s'est imposée très rapidement par la suite, comme I'a écrit Lady Eva Balfour en 1943 dans son ouvrage The living soil, sous l'inspiration de Howard: elle considéra rapidement "les santés du sol, des plantes et des hommes comme unes et indivisibles". Elle fut la fondatrice de la Soil Association en 1946. Howard a joué un rôle majeur dans le développement des concepts posant les bases des pratiques de l'agriculture biologique et de sa diffusion dans le grand public. La conséquence fut une "polarisation " des agricultures en bio et non-bio entre 1940 et 1978, période pendant laquelle le dialogue fut difficile entre les deux camps [6]. Dans le monde agricole, I'usage qualifie de "conventionnelle" l'agriculture non bio; ce 
terme révèle bien son aspect normé et majoritaire. Certains parlent aussi $d^{\prime}$ " agriculture industrielle et chimique" (référence à son mode d'organisation économique et sociale ainsi qu'à son orientation technique) [3]. Ces deux appellations mettent en exergue la polarisation entre deux conceptions de l'agriculture.

Cette polarisation ne fut pas à l'avantage de la bio puisque ses promoteurs furent suspectés de s'opposer au principe général de progrès en remettant en cause les apports de l'intensification. L'agriculture biologique, avec ses innovations agronomiques, fut rapidement désignée par la négative: une agriculture qui n'emploie pas d'intrants (engrais et pesticides) de synthèse. Et, malheureusement l'évolution des cahiers des charges est souvent allée dans le sens de prescriptions distinguant des produits autorisés de produits interdits. II en résulte qu'aujourd'hui encore les principes positifs à la base de l'agriculture biologique, qui représentent également sa marge de progrès, sont souvent méconnus du grand public et des décideurs [7]. À nos yeux, cette opposition perdure dans les compréhensions et approches différentes des systèmes de production et de la place de I'homme en leur sein. En effet, pour l'agriculture conventionnelle, l'objectif principal est toujours le rendement; tout est mis en œuvre pour l'atteindre et l'augmenter. La place de l'homme est alors très invasive : I'homme cherche à maîtriser au maximum le système en pourvoyant à presque tous les besoins des cultures. Les obstacles à l'atteinte du rendement sont éliminés ou contournés: la plante est malade, on applique un pesticide pour éliminer la maladie sans essayer d'en comprendre I'origine. Avec les pratiques de I'agriculture biologique, on cherchera à créer des conditions favorables pour une production optimale, en fonction du terroir, en quantité et en qualité par le maintien de la santé du sol et notamment en stimulant la vie dans le sol (micro-organismes et faune du sol). Si le système est en bonne santé, les maladies ne proliféreront pas ou peu.

Le regard sur la maladie est très révélateur des deux systèmes de pensée qui régissent le développement de l'une et l'autre agriculture. Matthieu Calame en fait une démonstration pleine de bon sens dans un ouvrage sur les perspectives de la bio [7] : dans la pensée soutenant l'agriculture conventionnelle, la maladie a une cause exogène, la faute à pas de chance et à l'attaque de parasites ou insectes dont il faut se protéger (vision que l'auteur désigne " bello-mécaniste ") et dans celle promouvant l'agriculture biologique, la maladie traduit un dysfonctionnement de l'ensemble de la pratique (sélection/variété cultivée, agencement de la parcelle/territoire, fumure, travail du sol...), les " bio-agresseurs " étant alors les « professeurs " de la nature.

Pendant des dizaines d'années, les sciences agronomiques au service du développement de l'agriculture conventionnelle ont produit des connaissances et élaboré les agrosystèmes pour maximiser la production d'aliments de base et de fibres, pour la consommation directe ou la transformation [8]. L'agriculture conventionnelle s'étant focalisée sur le rendement, elle en oublie que le système de production est une somme très complexe d'interactions entre les différents êtres vivants de l'écosystème et qu'en ne considérant que l'objet qui donnera du profit, on laisse de côté une multitude de collaborateurs, voire on empêche des collaborations qui pourraient être nécessaires et fructueuses. Le premier à avoir reconnu les limites de la conception " chimique" de l'alimentation des plantes est son concepteur lui-même, Liebig. Cette citation est reprise dans un ouvrage [9] de Jean Boucher, préfacé par Raoul Lemaire, dans un Précis scientifique et pratique de culture biologique: "Je confesse volontiers que l'emploi des engrais était fondé sur des suppositions qui n'existaient pas en réalité. Ces engrais devaient amener une révolution complète en agriculture. Le fumier d'écurie devait être complètement exclu et toutes les matières minérales enlevées par les récoltes remplacées par des engrais minéraux" ".

Olivier de Schutter, juriste belge, au terme de six années passées au poste de rapporteur spécial des Nations unies pour le droit à l'alimentation concluait sa mission par un rapport le résumant ainsi à un journaliste du journal Le Monde [10] : "Notre modèle agricole mondial est à bout de souffle ". II appelle à un changement de paradigme comme de nombreux auteurs l'écrivent aujourd'hui, qu'ils soient du monde agricole ou de la recherche.

Dans ce contexte de changement nécessaire des conceptions, l'agriculture biologique n'est pas la seule solution évoquée d'emblée, de plus en plus nombreux sont ceux qui invoquent l'agroécologie.

\subsection{Agriculture biologique et agroécologie}

La mutation est en marche. Si en termes de conception, les agricultures biologique et conventionnelle sont diamétralement opposées, les pratiques d'aujourd'hui se diversifient et montrent un gradient complexe sur lequel il est extrêmement difficile de mettre des bornes. Ainsi, certaines manières de faire de l'agriculture biologique en respectant le cahier des charges seront du type " agriculture conventionnelle " d'un point de vue conceptuel (on appliquera des pesticides, mais autorisés par I'AB). Inversement, il existe aussi des pratiques de l'agriculture conventionnelle qui introduisent un raisonnement proche de l'agriculture biologique quand, par exemple, I'agriculteur cherche à maximiser la vie de son sol même s'il n'a pas exclu tous les produits chimiques. Cette agriculture est banalement appelée "à bas intrants" (low input farming). Son objectif premier est de réduire les intrants tout en maintenant la productivité en quantité et la viabilité économique (figure 1).

On parle de "transition agroécologique". L'agroécologie a néanmoins un contour flou; son signifié recouvre des réalités différentes selon le contexte, les acteurs ou le continent considérés [11]. Le concept d'agroécologie a été développé avec les travaux de Altieri [12], dans son approche écologique des systèmes agricoles traditionnels pour gérer les ressources naturelles au bénéfice des plus démunis, confrontés à un système agroalimentaire de plus en plus mondialisé et industrialisé. Dans la dernière décennie, le nombre de publications et d'initiatives que leurs auteurs décrivent comme agroécologiques a augmenté de façon exponentielle [13].

Dans une vision large, I'agroécologie peut être définie comme l'étude intégrative de l'écologie dans l'ensemble du système alimentaire, intégrant les dimensions écologiques, économiques et sociales.

Au-delà du positionnement disciplinaire, son ambition est non seulement de transformer l'agriculture mais aussi de repenser I'ensemble des systèmes alimentaires afin de favoriser les transitions vers des systèmes répondant au développement durable [14]. C'est cet aspect de transition que le niveau politique a repris pour introduire l'environnement dans le devenir agricole français [15]. Des définitions minimalistes coexistent aux côtés de ces ambitions plus politiques, à l'image de celle que donne I'INRA (Institut national de la recherche agronomique) et qui se limite à une science aux confins de l'écologie et de l'agronomie.

Comment I'agriculture biologique trouve-t-elle sa place dans le mouvement agroécologique, quelle est sa particularité ? Pour certains auteurs, l'agriculture biologique est la mise en pratique de I'agroécologie : "Le consensus actuel est que l'agroécologie décrit une discipline académique étudiant les systèmes agronomiques, alors que l'agriculture biologique est l'application et l'intégration de la science avec l'expérience pratique des agriculteurs pour concevoir des systèmes productifs au champ et satisfaire à des crières de certification" ("Current consensus is that agroecology describes an academic field of systems study, while organic farming is the application and integration of science with practical farmer experience to design productive systems in the field and meet certification criteria ") [16]. Pour d'autres, il ne peut y avoir 


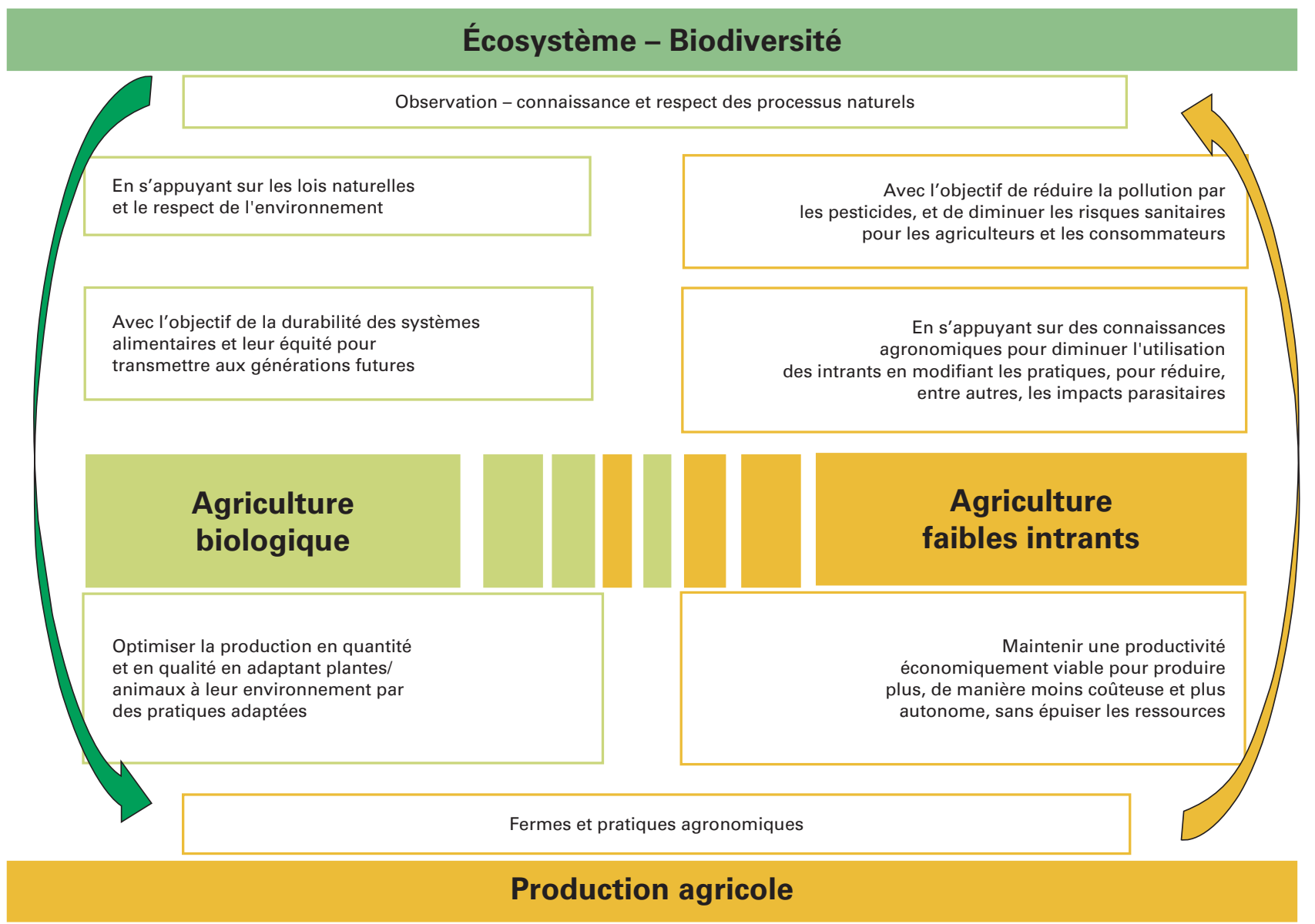

Figure 1 - Schéma de compréhension et stratégies des agriculture biologique et agriculture à faibles intrants : la première s'appuie sur une connaissance et le respect des processus naturels pour concevoir le système de production, la seconde essaie de rendre le processus de proconnaissance et le respect des processus nat
duction plus respectueux de l'environnement

confusion car l'agriculture biologique n'est pas toujours agroécologique, les pratiques biologiques dites " industrielles " n'ayant rien d'agroécologique et peuvent être tout aussi perturbatrices pour l'environnement que l'agriculture chimique intensive. De plus, Miguel Altieri [17] en dénonce des travers socio-économiques qui sont du même ordre que ceux de l'agriculture industrielle quand elle est pratiquée dans les pays du Sud avec des intrants, même biologiques, extérieurs à la ferme, pour des marchés étrangers et avec des coûts de certification élevés. Les marchés de niche pour les riches des pays du Nord posent les mêmes problèmes que tout système d'agro-exportation qui ne donne pas la priorité à la souveraineté alimentaire pour les agriculteurs du Sud.

Si on revient à la bio des pionniers, celle de Steiner, Rusch et Howard, elle commence par un positionnement culturel et social fort, de la même façon que Miguel Altieri pour l'agroécologie: " ceux qui adhèrent à l'agriculture biologique ont au plus haut point conscience d'une cohérence de leur pensée et d'un mode d'approche des problèmes qui les distinguent de l'agriculture industrielle, non pas par le refus d'un progrès en général, mais le refus $d^{\prime}$ 'un projet et d'une vision particulière du vivant et de sa gestion nés et développés en Occident " [12]. Si les pionniers de la bio en France se sont mobilisés face aux dégâts de l'agriculture chimique sur les sols et la santé des hommes, les pionniers de I'agroécologie, dans le sillage d'Altieri, cherchent à protéger l'agriculture traditionnelle et nourricière des pays du Sud, dévorée par les avancées de l'industrialisation de leur agriculture, par la révolution verte.

Pour synthétiser, l'agriculture biologique, cadre dans lequel nous inscrivons notre recherche, tient à la fois des fondements autour du respect du vivant des pionniers européens de la bio et des prérogatives écologiques et sociales de l'agroécologie.

Cette bio est parfois désignée de "bio paysanne", pour bien marquer sa différence avec une agriculture biologique qui se " conventionnalise " avec des techniques et des marchés semblables à l'agriculture industrielle. Mais existe-t-il une recherche spécifique pour cette agriculture? La recherche n'est-elle pas la même pour tous les systèmes de production ? Nous proposons de développer une recherche non seulement veillant à l'application des cahiers de charges, mais aussi pour stimuler en amont une vision positive de I'AB sur les traces des pionniers intégrant toutes les dimensions du mouvement. 


\section{Pour une recherche spécifiquement dédiée à I'AB}

\subsection{Quelle recherche agronomique pour l'agriculture biologique}

En 1840, un siècle avant Howard, commençait l'histoire de l'agriculture conventionnelle avec les travaux de recherche de Liebig.

"En 1840, la Section "Chimie » de I'association britannique "British Association for the Advancement of Science" ("L'Association Britannique pour le Progrès de la Science ") a demandé un rapport sur la chimie organique. Leur invité était Justus von Liebig. Le paragraphe d'introduction de son ouvrage sur la chimie organique pour son application à l'agriculture et à la physiologie, définissait la chimie organique, et pour beaucoup, le reste du texte aurait eu des conséquences très importantes sur les pratiques en agriculture et pour la nutrition des plantes. Le premier paragraphe établit : "L'objet de la chimie organique est de découvrir les conditions chimiques essentielles à la vie et au développement parfait des animaux et des légumes, et en général de comprendre tous les processus biologiques de la nature qui sont des mécanismes soumis aux lois de la chimie. " " [18].

En même temps, la recherche agronomique prend son essor et revêt la forme que nous lui connaissons aujourd'hui où tout doit être chiffré. Howard, pionnier de la bio, s'est aussi élevé contre cette conception de la connaissance en matière d'agriculture.

"La domination des résultats quantitatifs est une autre faiblesse de la recherche scientifique. Cela a profondément influencé la recherche agricole. En chimie et physique, par exemple, les relevés de précision sont omniprésents: ces sujets se prêtent à des déterminations exactes qui peuvent être enregistrées numériquement. Mais la croissance des plantes et l'élevage des animaux appartiennent à la biologie, un domaine où tout est vivant et qui est aux antipodes de la chimie et de la physique. Beaucoup de choses qui comptent pour le territoire, telles que la fertilité des sols, la texture, les pratiques culturales, la qualité des produits, l'épanouissement et la santé des animaux, la conduite des élevages, les relations de travail entre le maître et l'homme, "l'esprit de corps" de la ferme dans son ensemble, ne peuvent être pesées ou mesurées. " [2].

Ces deux pionniers étaient sur deux trajectoires de recherche totalement différentes. Ces chemins de connaissances ne pourront pas se rencontrer puisque fondés sur des regards sur la vie et la position de I'homme totalement opposés. Le malentendu perdure encore aujourd'hui, même si des tentatives de rapprochement existent, et se retrouvent dans I'histoire de la recherche agronomique en France.

Ce n'est qu'en 2000 que I'INRA reconnaissait à son tour (après le ministère de l'Agriculture et les institutions européennes) I'agriculture biologique après des décennies d'incompréhension. Les auteurs d'un rapport clé sur le sujet [19] s'accordaient sur la dimension historique de l'évènement. Les ambitions restaient modestes, tout en admettant l'intérêt de I'AB comme prototype d'agriculture durable. Elle se distinguait ainsi " des démarches de type agriculture "raisonnée" ou "intégrée", qui ne s'engagent qu'à utiliser de façon plus parcimonieuse les intrants de synthèse, et ne sont pas encadrées par des cahiers des charges collectifs unifiés. Ainsi définie par des obligations de moyens très restrictives, I'Agri- culture biologique se traduit par un ensemble de pratiques empiriques, inégalement maîtrisées techniquement et guère validées scientifiquement". L'ambition fut alors " d'intégrer l'agriculture biologique dans un contexte plus large et de ne pas créer un secteur de recherches à part mais plutôt d'insérer cette problématique dans des programmes plus larges, de favoriser sa prise en compte par des équipes ne travaillant pas directement sur l'Agriculture biologique".

Depuis lors, I'intégration de I'AB dans les programmes de recherche est restée modeste. Réduite à sa dimension scientifique, l'agroécologie est devenue récemment un "concept rassurant" pour faire entrer les défis environnementaux dans la recherche agronomique, en intégrant l'agriculture biologique dans cette notion fourre-tout. En 2014, le directeur scientifique d'Arvalis (Institut du végétal) [20] définit l'agroécologie ainsi : "un mode de production qui s'appuie sur les régulations biologiques et les fonctions écologiques offertes par les agroécosystèmes, en œuvre dans les parcelles et leur environnement... Concrètement, il s'agit donc de mobiliser l'ensemble des leviers disponibles et utiles, y compris les intrants de synthèse, pour atteindre cet objectif de multi-performances". Cette vision ne remet pas en cause le système de pensée et reste très éloignée de la bio.

Actuellement, la volonté affichée est d'intégrer une approche agroécologique [21] dans l'institution, considérée comme un de ses grands défis : "Dès 2010, I'Inra a identifié, dans son document d'orientation, l'agroécologie comme l'un de ses deux chantiers scientifiques prioritaires. Ces travaux doivent participer à déployer une recherche qui anticipe et accompagne les mutations de l'agriculture et contribue à une sécurité alimentaire et nutritionnelle durable au XXI ${ }^{e}$ siècle". Le point de vue de l'institution est que toute recherche est neutre et que les applications qui en découlent peuvent être mobilisées indifféremment par I'agriculture biologique ou l'agriculture conventionnelle; on parle de "recherche mobilisable " de I'une pour I'autre. Dans un rapport très controversé [22] du directeur scientifique actuel, Hervé Guyomard, I'idéal est de rapprocher les deux systèmes d'agriculture, comme I'indiquent les deux questions traitées :

Pour " déterminer les possibilités d'évolution de l'agriculture francaise vers des systèmes de production agricole plus durables, l'objectif était d'analyser les marges de progrès offertes par, d'une part, les systèmes de production dits "biologiques" et, d'autre part, les systèmes de production dits "conventionnels", au travers de deux questions:

- Comment rendre l'agriculture biologique plus productive et plus compétitive?

- Comment organiser la transition de l'agriculture conventionnelle vers une agriculture plus durable?

Ces deux questions ont été explorées en s'appuyant sur une grille commune d'indicateurs des performances productives, économiques, environnementales et sociales, et en mobilisant l'ensemble des connaissances disponibles sur les systèmes agricoles innovants proposant de nouveaux compromis entre ces différentes performances, tant en agriculture biologique qu'en agriculture conventionnelle $"$.

Le document d'Orientation 2010-2020 est très informatif sur la place de l'agriculture biologique dans le projet de recherche de I'INRA. Sur les 52 pages du document, elle n'est citée qu'une fois, dans un encadré de présentation du projet SOLIBAM [23], un programme européen que nous avons coordonné. Seule l'agroécologie est mise en avant en la concevant seulement comme le rapprochement des sciences agronomiques et écologiques [24]. Un paragraphe de ce document révèle bien la difficulté d'intégrer d'emblée les dimensions sociale et éthique de l'agroécologie des pionniers: "Tout en reconnaissant que la polarisation des recherches vers l'action pourra amener à considérer ses interfaces avec, et ses extensions vers, les sciences économiques et sociales, c'est la fertilisation croisée des disciplines écologiques, agronomiques et zootechniques qui sera visée en premier lieu comme source de nouveaux concepts et d'innovations". 
Ainsi, I'INRA étant la seule institution nationale scientifique spécialisée dans la recherche pour l'agriculture en France, il n'y a donc pas de spécialité agriculture biologique reconnue. Par contre, dans certains pays voisins en Europe, la recherche pour l'agriculture biologique s'est particulièrement développée grâce à la mise en œuvre d'une organisation spécifique, le plus souvent par des initiatives privées telles que FiBL (Forschungsinstitut für biologischen Landbau) en Suisse, le Louis Bolk Institute aux Pays-Bas, I'Organic Research Centre au Royaume-Uni, où I'AB est considérée dans toutes ses dimensions. Tous ces organismes sont aujourd'hui mondialement reconnus comme des spécialistes de l'agriculture biologique.

Notre point de vue de scientifiques est qu'il faut développer une recherche spécifique pour I'AB, basée sur ses principes initiaux, car nous considérons que les concepts fondateurs des agricultures biologique et conventionnelle sont fondamentalement différents. Nous partageons cet avis avec des chercheurs qui ont étudié le phénomène récent de "conventionnalisation de l'agriculture biologique [4] ", déjà évoqué précédemment. Dans ce mouvement d'inclusion de I'AB dans une logique de l'agriculture conventionnelle, il n'y a plus de remise en question du positionnement scientifique, la recherche "traditionnelle " de nos institutions n'a plus à changer ses méthodes mais juste son "objet " qui devient l'agriculture biologique. Ces méthodes sont essentiellement analytiques et se sont développées en privilégiant la théorie à la réalité, en rupture avec les démarches empiriques qui ont dominé jusqu'au $\mathrm{XVIII}^{\mathrm{e}}$ siècle [25].

Bernard Chevassus-au-Louis, directeur général de I'Institut de 1992 à 1996, invite aussi, lors d'une conférence en 2006, à la réflexion sur un système de recherche qui a répondu aux besoins du $\mathrm{XX}^{\mathrm{e}}$ siècle mais n'est plus adapté à ceux du $\mathrm{XXI}^{\mathrm{e}}$ siècle [26]. "De manière paradoxale, on peut considérer que l'on a opéré une "inversion" du processus d'innovation, c'est-à-dire que l'on est passé de la quête d'innovations adaptées aux modes de production existants à la mise en place de modes de production adaptés aux caractéristiques et contraintes des innovations!".

Les outils scientifiques ne représentent que les moyens de passer d'une question à sa réponse, mais ces outils ne sont pas universels et sont contingents des concepts et hypothèses qui définissent un cadre de travail. On ne fait pas une œuvre de Fra Angelico avec les outils d'un peintre en bâtiment.

La France n'est pas encore dotée d'un réseau institutionnel spécifiquement dédié à la recherche pour I'AB [27]. L'INRA, I'INSERM (Institut national de la santé et de la recherche médicale), le CIRAD (Centre de coopération internationale en recherche agronomique pour le développement), les chambres d'agriculture participent à la recherche pour I'AB avec des niveaux d'engagement très variés. Quelques structures isolées comme le GRAB (Groupement de recherche en agriculture biologique) font de la recherche spécifiquement dédiée à $I^{\prime} A B$, mais de manière localisée. Sur le plan national, les acteurs de cette recherche sont coordonnés par I'ITAB (Institut technique de l'agriculture biologique) qui fait encore peu de recherche en propre mais qui est amené à prendre une part importante dans ce domaine du fait de sa spécificité $A B$, puisqu'il est très proche de l'ensemble des acteurs sur le plan national et international. L'ITAB appartient aujourd'hui aux associations internationales de référence telles que ECO-PB (European Consortium of Organic Plant Breeding), ou I'IFOAM (créée en France en 1972, faut-il le rappeler !). Les chercheurs INRA ne sont investis qu'à titre personnel dans ces instances de référence puisque seules les structures dédiées à l'agriculture biologique peuvent en être membres.

IFOAM est un espace privilégié de définition des concepts liés à I'AB et de réflexion en matière de recherche. En 2013, cette organisation internationale s'est dotée d'un groupe de travail, TIPI (Technology Innovation Platform of IFOAM), avec des représentants de très nombreux pays (environ 80 institutions) pour renforcer la cohésion en matière de recherche [28] :
"Les voies durables vers l'innovation nécessiteront l'engagement de tous les acteurs dans une science axée sur l'approche multidisciplinaire. Une telle approche vise à : (1) donner un pouvoir d'autonomie aux zones rurales, (2) intensifier les processus éco-fonctionnels qui produisent des services alimentaires et écosystémiques, et (3) fournir de la nourriture pour la santé et le bien-être de tous. Le nouveau paradigme proposé par TIPI est fondé sur une approche globale du système, I'engagement des agriculteurs, chercheurs et autres praticiens dans une approche de co-innovation, et les technologies open access qui s'adaptent aisément aux conditions locales. "

II est précisé dans un des objectifs de la plateforme TIPI : " pour aider IFOAM à baser ses orientations politiques sur une bonne science" ("To enable IFOAM policies to be based on good science $")$.

Notre objectif est d'apporter notre pierre à l'édifice de cette « bonne science " pour l'agriculture biologique, en développant une approche différente de la recherche en agriculture « conventionnelle ».

\subsection{Agriculture de la vie : rester humble devant la complexité}

"Le pauvre animal palpite dans le filet et perd en se débat tant ses belles couleurs; et même si on réussit à l'attraper intact, le voilà quand même pour finir épinglé là, rigide et sans vie ; le cadavre n'est pas la totalité de l'animal, quelque chose d'autre en fait partie, partie principale, et en cette occurrence comme en tout autre, partie principale des plus principales : la vie... " Goethe [29].

Les systèmes vivants de la chaîne alimentaire sont d'une telle complexité que I'homme ne peut pas appréhender toute la finesse de leurs mécanismes intrinsèques: il essaie d'en comprendre certaines clés nécessaires à une action de production pour sa propre survie ; cette incursion dans l'agroécosystème induit une dynamique de rééquilibrage permanent.

Mathieu Calame [30], agronome, ex-président de I'ITAB (Institut technique de l'agriculture biologique) exprime de façon subtile le lien entre l'écosystème et sa fonction productive : " $\dot{A}$ l'instar des systèmes forestiers, le but recherché est celui de la constitution d'un équilibre productif de l'agrosystème, ce qui peut apparaître comme un oxymoron. La notion d'équilibre renvoie de fait à la stabilité dans le temps de l'écosystème qui ne doit pas s'appauvrir, à l'idée que ce système produit toutefois un excédent exportable et consommable par l'homme. L'écosystème est donc à la fois perçu comme stable et comme productif... Ainsi une agriculture biologique parfaite, et plus largement une agriculture durable, exporterait de la nourriture tout en maintenant intact et le taux de matière organique accumulé dans l'agrosystème et l'activité biologique de celui-ci. A contrario une agriculture non durable tend à produire en épuisant peu à peu l'agrosystème, en "décapitalisant". Dans certains cas, l'étape ultime de cette décapitalisation est le désert ".

Ainsi, l'agriculture biologique, dont le but est de produire des biens nécessaires à la vie des hommes, est basée sur la collaboration entre le grand nombre d'éléments qui composent l'écosystème et la maximisation des processus vitaux inhérents au lieu considéré, afin de permettre la stabilité du système. Tous les éléments qui le composent ont des fonctionnements différents et complémentaires, variant dans l'espace et le temps. La recherche en matière $d^{\prime}$ 'agriculture biologique essaie de comprendre comment collaborer avec cette diversité pour respecter l'équilibre du système pour assurer sa " durabilité ", et par conséquent pour respecter les potentialités d'un environnement donné et assurer la survie de I'homme lui-même. 
"Je ne sais qu'une chose, c'est que je ne sais rien", disait Socrate. C'est à cette attitude d'humilité que nous invite la complexité de la vie. Le chercheur, et avant tout le " chercheur bio ", devrait donc avoir un regard d'émerveillement qui cherche à comprendre la logique interne de l'agrosystème pour l'accompagner dans sa fonction de production, et non pas un regard dominateur qui veut tout connaître pour tout maîtriser, regard mécaniste et industriel que I'on retrouve dans l'approche conventionnelle.

Cette recherche apparaît avant tout comme un travail d'ingénierie : on aborde une question dans un agrosystème donné dont on inventorie les paramètres (environnement, pratiques, organisation sociale) et "non un problème dans l'absolu ou un agrosystème dans l'absolu... L'ingénieur détermine en quelque sorte à l'avance ce qu'il veut produire (un pont, un agrosystème ayant telle ou telle caractéristique), mais il ignore au début jusqu'où il devra aller, une part du chemin se fera en marchant, en réagissant aux premiers résultats. De ce point de vue, c'est un peu I'inverse de la recherche qui admet qu'elle ne sait pas ce qu'elle va trouver mais qui s'oblige à un protocole dont elle ne sort pas. En ingénierie, qu'importe le flacon pourvu qu'on ait l'ivresse " [7].

Restons avec Mathieu Calame qui a proposé une définition de I'ingénierie biologique qui est donc l'art de la recherche de solutions particulières adaptées à un problème particulier : la pertinence locale prime sur le caractère générique. L'ingénieur travaille sur de nombreux cas uniques, il ne travaille pas avec des "lois" mais des "cas ". Son expérience accumulée constitue petit à petit une démarche analogique : rapprocher des cas entre eux, ou des phénomènes à différentes échelles. II en découle un fonctionnement " jurisprudentiel " dans l'accumulation des connaissances où la résolution de chaque nouveau problème isolé enrichit le savoir-faire sans jamais apporter une réponse absolument définitive à un nouveau cas. II n'existe pas de modèle théorique prédictif ni des lois fondamentales pour répondre à tous les cas de figure possibles et imaginables. "Le mode de fonctionnement "jurisprudentiel" nécessite des outils de "typologie", comparables à un code de loi enrichi par la jurisprudence. En face d'un problème/système nouveau, le praticien commence par retrouver à quelle famille de systèmes le système étudié se rapporte, il recherche les diagnostics antérieurs. D'où l'importance des bases de données, des outils d'aide à la décision, des méthodes d'analyse clinique, des techniques de conduite de projet en partant des objectifs à atteindre " [6]. Dans une vision de la recherche ingénierique appliquée à la bio, Ton Baars définit la "recherche expérientielle " à partir de la réalité par l'accumulation de l'expérience sur des cas : "Elle offre une chance de développer une agriculture biologique à partir de multiples "bonnes pratiques" basées sur des projets transdisciplinaires, des études de cas et des séries de cas" ("It provides an opportunity to develop organic agriculture as multiple best-practices based on transdisciplinary projects, cases studies, and case series ") [31].

Face à cette complexité des systèmes vivants, il nous paraît inconcevable de les aborder d'un seul point de vue, donc à partir du regard d'une seule discipline. II devient ainsi essentiel d'associer différentes disciplines et différents types d'acteurs (scientifiques, praticiens, citoyens, politiques) pour réunir les regards et les concepts complémentaires afin de comprendre, autant que faire se peut, et gérer durablement, I'ensemble de la chaîne alimentaire depuis le champ de production jusqu'au produit fini.

\subsection{Recherche multi-acteurs et transdisciplinaire dans une approche globale}

Howard, dans son Testament agricole de 1943, ne voyait pas comment science et pratique pouvaient être dissociées et écrivait déjà : "Est-il rationnel pour un sujet comme l'agriculture de chercher à séparer science et pratique?" ("Is it rational in such a subject as agriculture to attempt to separate science and practice? "). II proposait simplement ce que les scientifiques redécouvrent aujourd'hui pour la mise en œuvre de recherche et de formation pour développer l'agroécologie: "Nous allons au-delà de l'apprentissage de nouvelles connaissances et compétences, convaincus que souvent "il y a un plus grand écart entre la connaissance et l'action qu'entre l'ignorance et la connaissance" " ("We go beyond learning new knowledge and skills, convinced that often "there is a larger gap between knowledge and action than between ignorance and knowledge" ") [32].

La recherche proposée pour développer une agroécologie au " sens fort et globalisant " se rapproche du terrain et des acteurs en associant souvent les mots-clés: recherche participative (ou multi-acteurs) et transdisciplinarité. Une revue récente de 2013, de chercheurs californiens [33], y ajoute le concept d'approche orientée pour I'action, "Agroecology as a Transdisciplinary, Participatory, and Action-Oriented Approach ", dans une conception de I'agriculture et du système alimentaire très proche de celle que nous encourageons dans une ingénierie pour l'agriculture biologique.

«Nous considérons que les approches transdisciplinaires sont celles qui évaluent et intègrent différentes origines de savoir, qui peuvent inclure des disciplines scientifiques ou académiques, aussi bien que différents types de systèmes de connaissances (par exemple, expérientiels, locaux, autochtones, etc.), et qui se basent sur des questionnements concrets. " [33]

Les pionniers de l'AB sont partis d'observations très fines du terrain, de la ferme, que certains ont considéré déjà comme un organisme vivant [34] en soi. Néanmoins, I'approche orientée vers l'action a encore une connotation descendante où la recherche trouve ses applications dans la pratique. La réalité de notre activité de recherche co-construite avec les acteurs nous invite à proposer une autre formulation, une " approche basée sur l'action ou la pratique " (Action-Based Approach), où le mot " action " recouvre un sens large: de l'observation à la pratique. C'est notre immersion au quotidien dans le système alimentaire du sol à l'assiette qui détermine le contenu des problématiques et des objectifs. Même si la recherche conduit à l'action, les questions et la façon d'expérimenter sont déjà une émergence de l'action.

Cette immersion dans le système dans lequel nous avons choisi de travailler a deux conséquences :

- l'organisation participative de la recherche devient alors une évidence. Nous participons tous à la réalisation du même objectif d'un développement agricole et sociétal que nous co-construisons par les savoirs et savoir-faire innovés ensemble ;

- une part de notre activité quitte à première vue le caractère objectif, attribué à toute activité scientifique. Or, la démarche n'est pas dénuée de rationalité puisque, dans son présupposé, nous appartenons à l'écosystème terrestre dans lequel il nous paraît prétentieux de s'extraire pour l'observer. Nous assumons cette immersion qui nous amène à évaluer en permanence la distance que nous prenons par rapport au système étudié, que nous soyons au microscope ou dans un groupe de travail.

L'approche participative a été développée d'abord dans les sciences sociales pour l'éducation, la médecine puis étendue notamment à l'agronomie, tous les domaines où le changement des pratiques est un objectif prioritaire. Cependant, les formes de recherches participatives sont variées même si l'objectif commun est de mieux ancrer la démarche scientifique dans la réalité des pratiques et donc de produire des connaissances associées à I'action. Les recherches participatives se distinguent des recherches "conventionnelles " par une "délocalisation du pouvoir " dans tout le processus, mais elles se distinguent aussi entre elles par différents degrés d'implication des partenaires. L'approche participative commence par des changements dans les relations entre les chercheurs et les autres acteurs impliqués. 
Pour affiner la description du positionnement du chercheur (et tout autre acteur participant aux recherches) tentant d'approcher la globalité d'un agrosystème, nous nous sommes aussi inspirés des travaux de Ton Baars dans lesquels il intègre des approches de sociologues comme Bawden. Par cette distribution des activités (figure 2) selon les quatre descripteurs holisme/réductionnisme et objectivisme/subjectivisme, il devient possible de visualiser les approches complémentaires du système et d'en comprendre la cohérence de l'approche globale que nous proposons. Dans un objectif concret (voir exemples au chapitre 4), nous montrons comment nous passons d'un carré à l'autre, avec des acteurs parfois différents d'un carré à l'autre et des niveaux de participation variables de chacun d'eux. La démarche de recherche comprend ainsi des phases successives et itératives avec des approfondissements analytiques et systémiques, considérant l'importance de l'ancrage territorial et des apports de connaissances variées et complémentaires.

Les études de Ton Baars [35] concluent que "La science expérientielle offre un processus de réconciliation philosophique important par lequel une synthèse des différentes approches de la recherche devient possible pour résoudre les problèmes de la vie réelle: quantitative et qualitative, subjective et objective, réductionniste et holistique, pratique et science" (" experiential science offers an important philosophical reconciliation process whereby a synthesis of different approaches to research becomes possible in solving real-life problems: quantitative and qualitative, subjective and objective, reductionist and holistic, practice and science ").

Ainsi, de la même manière qu'il existe des liens entre les éléments des systèmes vitaux, il nous faut, en recherche, créer des liens entre disciplines, acteurs et étapes du processus de transformation des produits agricoles en produits alimentaires. Par exemple, associer des agriculteurs, des meuniers, des boulangers, des consommateurs, des scientifiques de la génétique, de la meunerie, de la boulangerie, de la microbiologie, de la qualité sensorielle, dans un projet pour comprendre les enjeux de différents facteurs qui influencent le comportement du blé, de la pâte, du pain.

Nous proposons donc de développer une approche "globale" dans un sens qu'on pourrait aussi désigner d'approche " complète " ou " complexe ". II s'agit par exemple d'aborder chaque étape du processus de production du sol au produit fini dans I'assiette du consommateur, en associant un maximum de disciplines concernées pour comprendre chaque étape, mais aussi leurs interrelations les unes avec les autres dans toutes leurs dimensions biologiques, agronomiques, culturelles, sociales, économiques. Chaque discipline garde son approche analytique ou systémique. L'objectif de recherche reste le même tout au long du processus et pour tous les acteurs impliqués. La combinaison des résultats de chacune des disciplines discutés selon les résultats des autres, et de façon itérative, crée cette approche globale et transdisciplinaire car on va souvent au-delà de la juxtaposition des disciplines.

En conclusion, le développement de l'agriculture conventionnelle a été accompagné d'une recherche institutionnalisée qui $\mathrm{n}^{\prime} \mathrm{a}$ pas intégré les fondements de l'agriculture biologique malgré des initiatives dédiées à I'AB depuis 2000. L'arrivée du concept d'agroécologie a facilité l'affichage de la prise en compte des questions environnementales dans la recherche agronomique en associant une dimension écologique. Cependant, I'approche globale de la bio en recherche est encore à asseoir en intégrant les dimensions sociale et éthique, parties indissociables du concept. Elle s'appuie en premier lieu sur une conception spécifique du vivant, et de son respect, appelant à aborder la complexité de l'agroécosystème avec la coopération de tous les acteurs concernés en construisant une transdisciplinarité basée sur l'action, intégrant des disciplines complémentaires intervenant tout au long de la chaîne alimentaire.

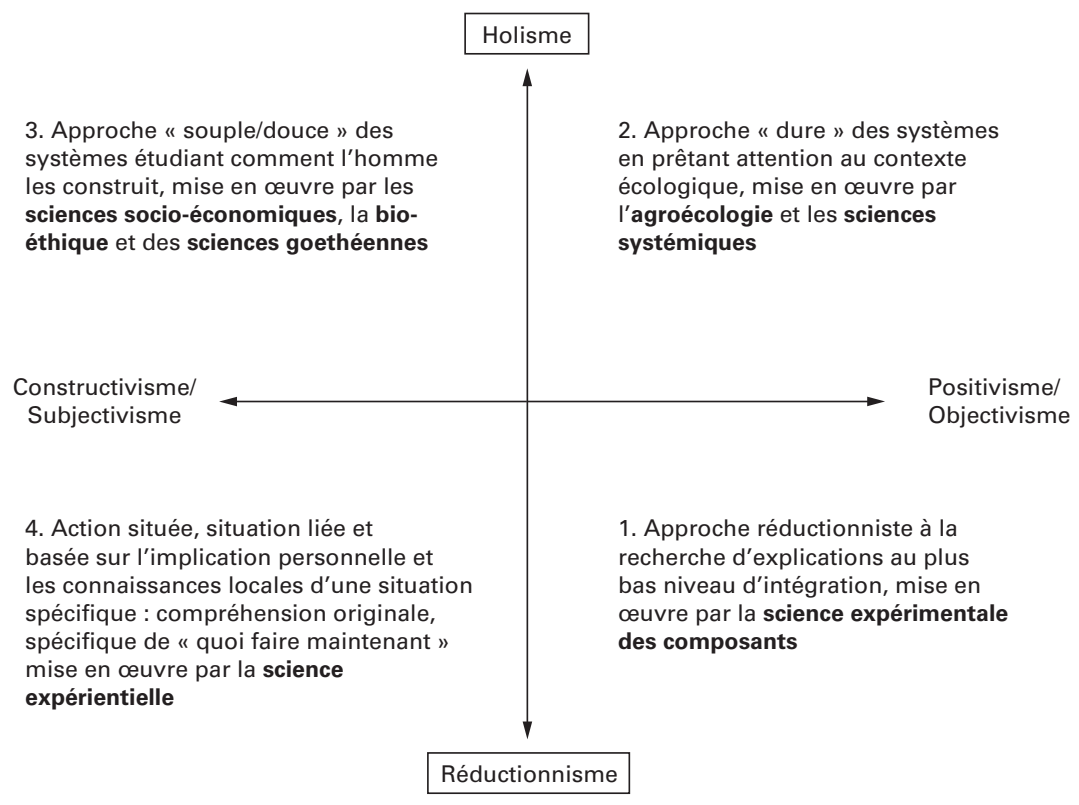

Figure 2 - Adaptation du carré de Bawden selon Ton Baars [35] pour positionner les actions de recherche pour l'agriculture biologique 


\section{Hypothèse : la diversité comme fondement d'une agriculture vivante}

\subsection{Diversités et agrosystèmes}

La biodiversité est reconnue comme essentielle à la vie sur Terre. Elle est aujourd'hui particulièrement menacée par les activités humaines [G 8 065]. Dans ce contexte, I'ONU avait déclaré l'année 2010 " année de la biodiversité " suivie, en 2011, par le lancement de la décennie pour inciter les États à prendre des mesures pour enrayer son érosion.

Les écosystèmes sont évolutifs. Le processus d'adaptation est un mécanisme permanent pour faire face au changement imprévisible. Dans ces conditions, la diversité est un élément clé de la stabilité à long terme et même de la survie. Cette hypothèse s'applique à la fois aux systèmes biologiques et économiques [36]. La diversité confère un potentiel de co-évolution aux systèmes vivants pour établir de nouvelles interactions en leur sein. L'agriculture, qui pourtant s'appuie fortement sur la diversité, participe à la dégrader considérablement dans les agroécosystèmes à trois niveaux: génétique (homogénéité génétique des variétés cultivées), spécifique (réduction du nombre d'espèces cultivées) et environnemental (à travers la simplification des pratiques homogénéisant les environnements).

L'agriculture biologique, telle qu'elle est proposée par les pionniers mais aussi telle que la FAO la décrit aujourd'hui [36], entend pallier ces tendances sur les trois niveaux en utilisant et développant des variétés locales ou hétérogènes, en mettant en œuvre des cultures associées, des pratiques et une gestion de l'environnement qui respectent et diversifient les conditions de culture.

Au niveau international, le concept de variété est défini par I'UPOV (Union internationale pour la protection des obtentions végétales) [38] et ouvre I'admission à un certificat d'obtention végétale :

"On entend par "variété" un ensemble végétal d'un taxon botanique du rang le plus bas connu qui, qu'il réponde ou non pleinement aux conditions pour l'octroi d'un droit d'obtenteur, peut être:

- défini par l'expression des caractères résultant d'un certain génotype ou d'une certaine combinaison de génotypes;

- distingué de tout autre ensemble végétal par l'expression $d^{\prime}$ au moins un desdits caractères, et ;

- considéré comme une entité eu égard à son aptitude à être reproduit conforme"

En France, le GNIS (Groupement national interprofessionnel des semences et plants) apporte des précisions dans ce cadre et définit la variété comme une " population artificielle obtenue en vue de son usage en agriculture, reproductible, homogène et stable dans ses caractéristiques génétiques et distincte des autres variétés " [39].

L'élargissement de la diversité du champ cultivé commencera par considérer celle de la semence, l'intrant initial de toute production agricole. Pour une production donnée, nos recherches commencent le plus souvent par étudier la diversité génétique de l'espèce considérée, celle qui existe au sein d'une même espèce (la diversité variétale) et celle qui existe au sein d'une même variété (la diversité intra-variétale). Pour optimiser l'agroécosystème, nous intégrons d'autres niveaux de diversités, comme la diversité spécifique (com- binaison de différentes espèces au sein d'une parcelle) et la diversité des pratiques culturales dans une dynamique pluridisciplinaire. La diversité des disciplines éclaire les caractéristiques complémentaires à chaque étape, du sol au produit.

Cette approche nous fait partir à l'opposé de la tendance normative et homogénéisatrice à l'œuvre depuis le siècle dernier qui intensifia son agriculture sur un modèle passe-partout basé sur les intrants chimiques. Néanmoins, notre démarche est loin d'être en marge des connaissances scientifiques récentes qui confortent toujours plus les intuitions et les choix des pionniers. Nombreuses sont les connaissances récentes en matière de mécanismes biologiques, génétiques et écologiques qui associent santé des organismes, résilience des agroécosystèmes et qualité des aliments à la diversité.

\section{2 Évolution de la place de la diversité dans l'agriculture}

\subsubsection{La diversité : clé de la stabilité des écosystèmes}

La diversité des cultures (intra et inter-variétale) a été identifiée comme un facteur clé pour leur résilience dans le contexte du changement climatique [40] [41], pour atténuer les variations environnementales plus marquées, la sécheresse [42] ou faire face à de nouvelles maladies [43] [44] [45] [46]. Au-delà de la parcelle agricole, la diversité des cultures joue un rôle favorable dans le maintien d'autres services écosystémiques que procurent la biodiversité sauvage associée aux agroécosystèmes [47] [48] [49]. Chez les céréales, les effets positifs de la diversité sous différentes formes (mélanges de variétés, composites cross populations... voir encadré 2) sont mentionnés dans de nombreuses publications [50] [51] [52] [53] et la capacité adaptative des populations évoluant avec leur environnement a déjà été décrite sur des critères phénotypiques et génétiques en relation avec les pratiques des agriculteurs [54] [55].

"La résilience est la capacité d'un écosystème à surmonter une perturbation en récupérant rapidement pour revenir à un état d'équilibre. Un système résilient va se réorganiser lors d'une perturbation, de manière à conserver l'essentiel de sa fonction, de sa structure, de son identité et de ses réactions. Ainsi, la résilience est liée aux capacités d'adaptation d'un système face à un changement " [56].

Les services écosystémiques sont "les bénéfices que les humains retirent des écosystèmes sans avoir à agir pour les obtenir. Cela inclut la mise à disposition de nourriture, d'eau, de bois, de fibre; des services de régulation qui affectent le climat, les crues, les maladies, les déchets et la qualité de l'eau; des services culturels qui procurent des bénéfices récréatifs, esthétiques et spirituels; ainsi que des services de support tels que la formation de sol, la photosynthèse et les cycles des nutriments " [57].

\subsubsection{Histoire de la sélection : révélatrice de la disparition de la biodiversité dans I'agriculture}

Les hommes ont commencé par domestiquer les plantes à partir de populations sauvages. Les populations cultivées se sont différenciées et diversifiées grâce au travail des paysans. Depuis le $\mathrm{XIX}^{\mathrm{e}}$ siècle, ce sont les entreprises semencières et des scientifiques qui ont pris le relais, dont la famille de Vilmorin en France, avec une homogénéisation des plantes cultivées et l'amélioration de la productivité. Avec le $\mathrm{XX}^{\mathrm{e}}$ siècle, les méthodes de sélection des plantes se sont affinées grâce aux connaissances apportées par la découverte des lois de la génétique. 


\section{Encadré 2 - Diversité des variétés étudiées et développées pour l'agriculture biologique}

La notion de variété contient en elle-même une grande diversité de formes selon la biologie, la génétique, I'histoire et l'usage. Selon la diversité génétique des différentes formes (ou types) de variétés utilisées et nouvellement développées pour l'agriculture biologique, on peut proposer un classement par ordre de diversité génétique croissante [61] :

Lignées pures : toutes les plantes sont identiques; la diversité génétique est très faible. Développées pour l'agriculture conventionnelle par la filière semences, c'est une forme de variété commerciale très courante pour les espèces autogames.

Variétés anciennes, "ancêtres" des lignées pures: toutes les plantes sont quasiment identiques (le niveau d'homogénéité est moins rigoureux que pour les lignées). Ce sont les variétés développées par les premiers semenciers à l'initiative de la filière semences. Elles ne sont aujourd'hui commercialisées que pour des plantes légumières, mais sont le plus souvent conservées dans des banques de graines ou maintenues par quelques passionnés.

Landraces ou populations locales: les plantes se ressemblent beaucoup et ont une grande partie de leur patrimoine génétique en commun pour les espèces autogames mais sont très diversifiées pour les allogames, tout en ayant des caractères, notamment de précocité ou qualité. Ces variétés ont été sélectionnées par des agriculteurs pour s'adapter à des conditions locales dans l'agriculture d'avant l'industrialisation et sont aujourd'hui sollicitées par certains agriculteurs bio. Tout comme les variétés anciennes, elles ne sont plus commercialisées et trouvent difficilement leur place dans la réglementation.

Mélanges variétaux ou populations dynamiques : ce sont des mélanges de plusieurs variétés (quel que soit leur type) dont la récolte est ressemée d'une année à l'autre. La diversité entre les plantes est donc grande et elle donne aux agriculteurs un fort potentiel de sélection et d'adaptation. Souvent spécifiques à la ferme qui les a créés, ces mélanges commencent à être étudiés dans le cadre de projets de recherche participative mais ne peuvent être commercialisés du fait de leur instabilité et de leur hétérogénéité.

Croisement bi-parentaux : c'est la descendance cultivée d'un croisement entre deux plantes. La grande diversité créée (qui diminue au cours des cycles de multiplication) offre une bonne potentialité de sélection pour les agriculteurs. Cette pratique est développée dans des projets de recherche participative pour les espèces autogames (populations créées par les chercheurs sur les suggestions des agriculteurs, puis dont l'évolution adaptative est ensuite suivie dans différentes fermes).

Composite Cross Populations (CCP) et mélanges de croisements : c'est la descendance cultivée de plusieurs croisements entre divers parents parfois réalisés sur plusieurs années pour maximiser les recombinaisons génétiques. La diversité entre les plantes est immense et reste très importante au fil du temps. Cette forme de variété est née à l'initiative de chercheurs pour l'agriculture bio dans l'objectif de maximiser la diversité pour avoir un potentiel d'adaptation (accompagné de sélection ou pas) important dans des conditions variées.

\section{Sélection et amélioration des plantes}

"L'amélioration des plantes est l'ensemble des activités tendant à l'ajustement génétique des plantes au service de I'homme et comme la réalisation de multiples adaptations aux milieux physique, biologique et économique " [58]

"L'amélioration des plantes est l'art et la science de la création de variétés ayant des caractères bien définis " (André Gallais, professeur émérite d'AgroParisTech, spécialiste de génétique quantitative et des méthodes $d$ 'amélioration des plantes [59]).

Un groupe d'experts [60] regroupés par la FRB (Fondation pour la recherche pour la biodiversité) pour faire un état des lieux de la diversité cultivée ont récemment montré la réduction très importante de cette diversité. La première étape a été la conception d'un indicateur ad-hoc prenant en compte plusieurs facteurs d'homogénéisation (comme l'homogénéisation génétique, par disparition de la diversité à l'intérieur des variétés, ou spatiale, avec des départements cultivant de plus en plus les mêmes variétés).

L'exemple d'une espèce très importante comme le blé est très pédagogique (figure $\mathbf{3}$ ). Il subit une homogénéisation globale de la diversité génétique des variétés cultivées en France entre 1912 et 2006: " une homogénéisation de la diversité génétique à l'intérieur des variétés avec le passage de "variétés populations" à des lignées génétiquement pures, surtout observé entre 1912 et 1964 ; puis une uniformisation de la diversité génétique entre les variétés majoritairement cultivées qui tendent à être de plus en plus proches génétiquement dans le temps: ce phénomène est particulièrement vra depuis le début des années 1980 ; et enfin une homogénéisation de la diversité entre départements où l'on cultive de plus en plus les mêmes variétés : ce phénomène est particulièrement observé depuis le début des années 1990 ". Enfin, même si le nombre de variétés tend à augmenter, elles présentent une plus forte proximité génétique entre elles.

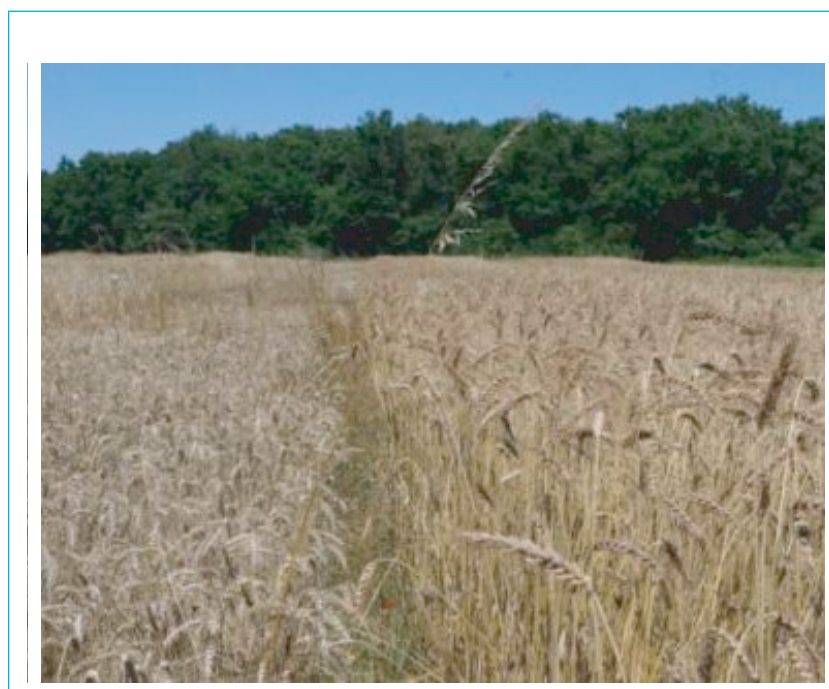

Figure 3 - Essai à la ferme en conditions bio : à gauche, une variété moderne homogène et à paille courte, à droite une population paysanne diversifiée et à paille haute 


\subsubsection{Visions en opposition jusqu'à la conception de la variété cultivée}

Jusqu'au XIX ${ }^{\mathrm{e}}$ siècle, le travail de sélection était assuré par les communautés paysannes et inséré dans le fonctionnement des fermes. L'univers de la semence a basculé en très peu de temps. Le monde de la sélection et de la semence est aujourd'hui organisé, de la recherche à la réglementation, sur une hypothèse de base : la variété cultivée est un input agricole stable et homogène, et régie par un système de protection intellectuelle (encadré $\mathbf{3}$ ).

La division du travail propre à toute production industrielle s'est imposée à la semence au $\mathrm{XX}^{\mathrm{e}}$ siècle, avec la création de métiers différents pour la création variétale, la multiplication des semences et la simple production agricole: les métiers de sélectionneurs (privés ou publics), de multiplicateurs de semences et d'agriculteurs consommateurs de semences sont devenus complètement distincts. La variété homogène et stable est proposée comme essentielle au développement de l'agriculture "moderne". Bonneuil et Thomas [62] font une rétrospective historique précise de cette mutation profonde de notre agriculture en citant un chercheur, futur directeur de I'INRA dans les années 1940, qui définit la variété "du point de vue du phytotechnicien et de l'agronome". Celui-ci distingue trois types de variétés : "la variété lignée pure, la variété clone et la variété population " et il privilégie I'homogénéité comme garante de la prévisibilité et stabilité de la valeur agronomique et technologique d'une variété. Les variétés populations, aussi désignées variétés paysannes ou locales, sont " susceptibles de varier dans l'espace et dans le temps". En parallèle de cette vision, les méthodes expérimentales sont restreintes aux stations d'expérimentation et quittent les champs des agriculteurs. Aujourd'hui, cette conception "fixiste " de la variété domine toujours la recherche et la réglementation [63].

Cependant, de nouveaux besoins en termes de variétés émergent avec le développement de l'agriculture biologique, et toute agriculture cherchant à diminuer les intrants. Le rôle de la variété cultivée dans la performance de l'agroécosystème est alors reconsidéré avec, en parallèle, la mobilisation d'écologues, généticiens, botanistes, agronomes pour sensibiliser le monde agricole et les décideurs aux risques de la perte de la biodiversité sauvage et cultivée: "La vie c'est comme une bicyclette, il faut avancer pour ne pas perdre l'équilibre " disait Albert Einstein. Et comme le précise Pierre-Henri Gouyon: "la biodiversité, c'est un système dynamique, on ne la conservera que si on laisse le fonctionnement de ce système exister. La crise de la biodiversité actuelle est une crise globale du fait que le moteur est en panne. Cela se voit surtout dans le monde agricole où on sème des millions $d^{\prime}$ hectares avec les mêmes variétés. Cela pourrait créer des famines " [65].

La diminution de la diversité au sein de l'agriculture, et notamment du point de vue génétique, va de pair avec son industrialisation. Or, l'agriculture biologique, celle que nous avons qualifiée "bio paysanne" (voir fin §1.2), qui se distingue par essence de cette vision industrielle, appelle un redéploiement de la diversité à tous les niveaux pour trouver une productivité qui respecte les équilibres des écosystèmes.

\subsection{Nécessité de redéployer la diversité pour la bio paysanne}

Bertrand Hervieu, président de I'INRA de 1999 à 2003, faisait des constats - toujours d'actualité - sur la relation entre la culture scientifique inhérente à la sélection et la rupture avec les processus naturels d'une part, et la rupture avec la société paysanne, d'autre part: "Ce processus d'amélioration des plantes a instauré et diffusé dans le corps social une culture scientifique marquée par une sorte de distanciation, d'éloignement et même de rupture vis-à-vis de la nature; cela afin de la connaître, la transformer et I'utiliser. II s'agit là d'un processus banal, inhérent à toute démar-
Encadré 3 - La réglementation appliquée aux variétés et semences

Dans le monde, deux systèmes semenciers fondamentalement différents se côtoient [64] : (1) le système formel réglementé, caractérisé par une chaîne d'activités qui va de I'amélioration des plantes à la diffusion de semences auprès des agriculteurs (avec une organisation top-down) et (2) le système informel, dont les activités relatives aux semences sont organisées au niveau local, intégrées au processus de production que les agriculteurs conservent ou diffusent dans un cercle d'échange restreint (organisation en réseau).

Il existe donc deux grandes catégories de semences: les semences "certifiées " issues des semenciers professionnels et les semences paysannes qui circulent dans les systèmes informels. La majorité des semences dans le monde sont produites par les paysans eux-mêmes et représenteraient les $2 / 3$ de toutes les semences utilisées au niveau mondial, notamment dans les pays du Sud.

Le système formel est réglementé afin de (1) conserver l'identité et la pureté des variétés, et (2) garantir la qualité physique, physiologique et sanitaire des semences. La législation actuelle de I'UE pour la commercialisation des semences repose ainsi sur deux processus : (1) l'enregistrement des variétés sur un catalogue officiel et (2) la certification des semences selon plusieurs directives européennes établies par espèce ou groupe d'espèces. II faut ajouter un troisième volet de la réglementation assurant la protection de la propriété intellectuelle: celle de l'obtenteur qui a été créée par la convention internationale UPOV, adoptée à Paris en 1961. Elle définit les droits de propriété appelés " certificat d'obtention végétale " (COV) au sélectionneur. Contrairement au brevet, le COV ne peut pas interdire l'utilisation de la variété protégée pour en sélectionner une autre. Les semences paysannes ne sont pas protégées par des droits de propriétés intellectuelles.

En Europe et où le système UPOV est appliqué, la commercialisation des semences passe par l'inscription à un catalogue officiel des variétés. Les critères d'inscription des variétés sont de deux ordres: (1) critères DHS, les mêmes exigés pour l'obtention du COV (Distinction - avec les autres variétés -, Homogénéité, Stabilité - la distinction permettant de garantir un titre de propriété) et (2) de bonne "valeur agronomique et technologique " (VAT), pour répondre aux critères de l'agriculture dominante, de la transformation industrielle et de la grande distribution. Pour les grandes cultures, on ajoute parfois un critère environnemental (on parle alors de "VATE ").

Depuis 2008, des réflexions ont été engagées au niveau de la Communauté européenne dans une démarche nommée "Better Regulation", qui consiste en la révision du corpus règlementaire actuel relatif à la commercialisation des variétés, des semences et des plants. Cette révision entend prendre en compte les différentes origines de semences présentes dans le paysage européen pour répondre aux demandes des utilisateurs des semences paysannes, biologiques ou " anciennes", pour (1) reconnaître le droit des agriculteurs à échanger leurs semences, (2) pour faciliter l'enregistrement des variétés anciennes et de "variétés hétérogènes" non conformes aux standards DHS du catalogue et (3) autoriser les micro-entreprises à commercialiser des semences de variétés "de niche " non enregistrées. Cette proposition a été rejetée par le Parlement. Le processus de révision est toujours d'actualité.

che scientifique. La particularité de l'amélioration des plantes est que ce phénomène s'est heurté à une vision de la nature héritée des sociétés paysannes. La France - à la différence d'autres pays, notamment du Nord de l'Europe - est en effet un pays qui a mis longtemps à faire le deuil de son identité paysanne; un pays où l'état de paysan a cédé la place au métier d'agriculteur à la fois tardivement et brutalement: n'oublions pas qu'il y a moins d'un demi-siècle les agriculteurs représentaient près d'un tiers de la population active contre un peu moins de $4 \%$ aujourd'hui ! " [66]. 
Or, aujourd'hui des études sociologiques révèlent une forme de renaissance paysanne, observée au niveau international. Jan Douwe van der Ploeg [67], de I'université de Wageningen, explore la position, le rôle et l'importance de la paysannerie dans un contexte de mondialisation, en particulier celui des industries agroalimentaires et des produits alimentaires. La condition paysanne est caractérisée par une lutte pour l'autonomie par la création et le développement de ressources locales et des pratiques associées au développement durable.

L'agriculture paysanne est fondamentalement différente de la vision entrepreneuriale et intensive de l'agriculture conventionnelle. Il démontre que les paysanneries de ce monde sont loin d'être en déclin et sont une solution pour revaloriser les terroirs.

L'ancrage local, territorial et culturel de l'agriculture biologique est intrinsèque à sa nature. Pour bien marquer cette particularité, les agriculteurs bio préfèrent souvent être qualifiés de "paysans". Cet attachement à la paysannerie fait souvent la différence entre une agriculture biologique tournée vers le marché et une bio héritée des pionniers. Nature \& Progrès [68] en fait un de ses objectifs : " une agriculture biologique qui préserve le tissu rural et le métier de paysan en le revalorisant "; un autre montrant la cohérence entre la conception du métier de paysan et la semence: " pour une agriculture biologique qui soutient les savoir-faire et les semences paysannes".

En somme, l'agriculture conventionnelle, et la sélection végétale qui lui a été dédiée, ont été conçues sur la base de l'homogénéité des peuplements végétaux. Or, la diversité à différents niveaux, et spécifiquement dès la semence, est indispensable au développement d'une agriculture biologique et paysanne pour une stabilité de la production et des écosystèmes. Ainsi, la diversité est l'hypothèse centrale de notre démarche de recherche.

Par une recherche proche des acteurs et pluridisciplinaires, nous nous attachons à prolonger le travail des pionniers de l'agriculture biologique avec des "paysans bio", attachés à la fois : (1) à renforcer l'efficacité des processus biologiques et écologiques de leurs agroécosystèmes par des plantes et des pratiques adaptées, et (2) à valoriser les qualités et la diversité des produits en marquant leur accroche au terroir et aux valeurs culturelles.

\section{Notre métier : la diversité des savoirs au cœur du processus de recherche}

\subsection{Au cour de nos travaux : la biodiversité cultivée}

\subsubsection{Manque de variétés adaptées à l'AB : rencontre entre agriculteurs et chercheurs}

La diversité variétale a été la question initiale et le fil rouge de tous nos travaux de recherche. La semence est un élément clé dans les systèmes agricoles et l'histoire des semences et de la sélection végétale est à l'image de la société elle-même.
L'agriculture s'est mise au diapason de la révolution industrielle de l'agriculture avec la séparation des métiers de l'agriculture.

"Dans cette crise, la question de la relation à la nature et au vivant, qu'il soit animal ou végétal, est pourtant essentielle. Or, la recherche n'a pas su y apporter un minimum d'explications et encore moins de réponse, alors même que la science portait une lourde responsabilité dans le bouleversement de la conception du vivant. On a mis la science du côté du progrès en oubliant qu'elle était aussi synonyme d'arrachement et de transformation de notre identité. Au final, c'est le deuil inachevé de cette identité perdue qui suscite des tensions très fortes aujourd'hui. Tout cela n'est pas l'écume des jours ou le clapotis des vagues : nous sommes en face de problèmes extraordinairement profonds qui sont loin d'être réglés" [66] constatait Bertrand Hervieu, président de I'INRA lors de l'introduction d'un colloque à Montpellier à I'occasion des 40 ans du département de recherche "Amélioration des Plantes".

La semence, dont toute l'information génétique (et épigénétique) qu'elle porte constitue les caractéristiques de la variété, est une forme de mémoire des conditions environnementales locales (sélection naturelle) et du travail de I'agriculteur (sélection intentionnelle). Dans un contexte intensif les conditions sont standardisées alors que, dans un contexte biologique, elles sont uniques. Le rôle de la semence est alors d'autant plus stratégique que son choix et sa sélection représentent un moyen de valoriser les spécificités de l'environnement.

Le manque de variétés pour l'agriculture biologique s'est fait sentir au moment de la mise en application d'un règlement européen imposant de produire $A B$ avec de la semence $A B$ [69]. Celui-ci est sorti en 1995 mais son application a commencé en 2004 , avec la mise en place d'un site officiel dans chaque pays de la Communauté européenne. L'évolution du marché de la disponibilité des semences biologiques s'observe sur l'évolution du nombre de dérogations demandées par les bio pour utiliser des semences non $A B$, rendant compte des indisponibilités de semences bio sur le marché. Une étude réalisée dans le cadre du programme SOLIBAM [70] a montré que la grande majorité des producteurs biologiques utilisent volontiers des semences certifiées $A B$ pour assurer l'intégrité du secteur (encadré 4). Sauf pour les cultures fourragères et quelques espèces végétales, la disponibilité et l'utilisation de semences biologiques se sont considérablement améliorées depuis 2010, avec la diminution du nombre total de dérogations en France depuis 2012.

Cependant, le nombre total de dérogations reste très élevé en céréales notamment, avec une utilisation de seulement 45 à $70 \%$ des semences biologiques. Pour les légumes, les semences $A B$ sont plus disponibles. En effet, $82 \%$ des agriculteurs sont des utilisateurs réguliers de semences biologiques, même si parfois ils utilisent jusqu'à 70 variétés différentes. Une information apportée par une enquête complémentaire, réalisée auprès de 118 producteurs de céréales, sur des pratiques très variées (depuis la polyculture-élevage jusqu'aux paysans-boulangers) révèle que $83 \%$ d'entre eux utilisent des semences de variétés populations.

L'utilisation de ce type de variétés a augmenté au cours des cinq dernières années, notamment grâce à la mise en œuvre de programmes de sélection participative et essais techniques suite à la création du Réseau semences paysannes en 2003. Au-delà de la question économique relative au coût (seulement $29 \%$ d'entre eux évoquent cet argument pour l'utilisation des populations), les paysans cherchent d'abord des semences adaptées à leur environnement de production (44\%) et à asseoir l'autonomie dans leur système de production ( $33 \%$ ). L'enquête de SOLIBAM a révélé leur intérêt pour la qualité $(20 \%)$ et pour la biodiversité $(16 \%)$. Les agriculteurs interrogés mettent en avant la diversité génétique intrinsèque des populations et leur capacité à évoluer et à s'adapter aux différents sols, conditions climatiques et pratiques culturales. 


\section{Encadré 4 - Disponibilité en semences biologiques} (SOLIBAM)

L'étude réalisée dans le cadre du projet de recherche européen SOLIBAM (2010-2014) a révélé plusieurs facteurs limitant I'utilisation de semences biologiques et de l'offre [70] :

- un manque de rentabilité pour les semenciers : les coûts de production sont beaucoup plus élevés que pour la production de semences conventionnelles en raison de rendements de semences biologiques inférieurs $(50 \%$ de moins pour le blé, l'orge, le maïs et les fèves); c'est un marché fragmenté (niche) avec une demande très diversifiée par rapport aux semences conventionnelles :

- un prix élevé des semences biologiques pour les producteurs comparé aux semences non traitées (30 à $100 \%$ selon les espèces) : rendement de semences biologiques deux fois moins élevé que celui des conventionnelles ;

- un manque de disponibilité de variétés adaptées, en particulier pour les cultures fourragères et légumières, dû (1) aux difficultés de multiplication (plantes inadaptées à la conduite bio et manque d'agriculteurs biologiques prêts à multiplier les semences), et (2) aux stratégies d'entreprises non favorables à une filière de semences biologiques ;

- un manque de connaissance des semenciers professionnels des besoins et des attentes des agriculteurs biologiques ;

- un nombre important de contrôles auxquels les semences biologiques doivent se conformer (plusieurs règlements), décourageant pour les professionnels: (1) enregistrement des variétés sur le catalogue commun (directive 2002/53, directive $2002 / 55)$, (2) réglementation sur la qualité des semences et (3) certification selon le cahier des charges de l'agriculture biologique (selon les directives du Conseil 834/2007 et 889/2008) ;

- un très faible investissement dans la sélection végétale biologique compte tenu du faible marché, de l'inadaptation de I'enregistrement au catalogue et du coût de la procédure.

Le manque de semences $A B$ est donc encore d'actualité et l'application du règlement imposant de produire $A B$ avec des semences $A B$ s'est fait attendre faute de semences. Dans les années 2000, des agriculteurs interpellent des chercheurs pour aborder le problème. L'INRA, représentant la recherche publique en France, a été incité à s'intéresser aux besoins de l'agriculture biologique à cette même période. Un comité interne pour l'agriculture biologique (CIAB) a été créé et doté de fonds pour soutenir les premiers projets en 2000. L'un d'eux [71] (2001-2003) posait la question des producteurs bio de choux et choux-fleurs en Bretagne. Le contexte était favorable car: (1) les producteurs avaient une organisation collective (IBB, InterBioBretagne), (2) I'INRA disposait de ressources génétiques collectées dans les années 1980 sur des fonds européens pour sauvegarder les variétés locales et paysannes des espèces cultivées en Europe et (3) de nombreuses variétés modernes de choux étaient incompatibles avec les principes de I'AB suite à I'introduction de biotechnologies dans le schéma de sélection. L'objectif de ce projet était de jeter les bases d'une sélection adaptée aux besoins des producteurs en les impliquant dès le départ. Le remplacement des variétés traditionnelles par des variétés hybrides était récent (premier hybride au début des années 1980) et les paysans bretons disposaient encore des savoir-faire en matière de production de semences à la ferme. C'est spontanément qu'ils se sont proposés pour multiplier les variétés populations issues de ressources génétiques, présentant, après évaluation collective, les critères requis pour la production. L'ingénieur chargée du projet a accompagné les agriculteurs sur les observations à réaliser et complété les connaissances nécessaires pour la multiplication. Surtout, elle a élargi le panel des ressources génétiques à explorer, en recherchant des variétés au-delà des populations traditionnelles bretonnes, en fonction des connaissances sur I'histoire de l'espèce qui $n$ 'est devenue " traditionnelle » en Bretagne qu'à la fin du XIX ${ }^{e}$ siècle. Pour certains types de choux, des croisements ont été réalisés parallèlement par des techniciens en sélection végétale et un employé de I'organisation collective des producteurs. Ainsi est née la sélection participative en Bretagne. Ensuite, pour tenir compte du cadre réglementaire inadapté aux échanges de semences de variétés non inscrites (ce qui est le cas des ressources génétiques multipliées et sélectionnées par les paysans), les paysans ont créé une association, "Kaol kokh" ("vieux chou " en breton), pour donner un statut collectif aux semences et les échanger, ainsi que les savoir-faire associés.

En même temps que l'expérience sur les choux commençait en Bretagne, d'autres groupes d'agriculteurs se formaient autour de chercheurs de I'INRA et ingénieurs-animateurs d'associations de producteurs en 2001-2002 autour du blé dur en Camargue, du maïs en Aquitaine, de la tomate dans l'Aude. Les premiers groupes de travail multi-acteurs de la sélection participative française étaient nés. Des paysans-boulangers pionniers avaient commencé dès la fin des années 1990 à réintroduire dans leurs champs des "variétés anciennes" de blé (souvent qualifiées de "blés anciens ") dont la farine est plus adaptée à la fabrication de pain traditionnel au levain naturel. En 2003, tous ces paysans se retrouvaient à Auzeville, près de Toulouse, pour créer le Réseau semences paysannes [72].

\subsubsection{Questions spécifiques pour chaque espèce}

Ce travail de recherche collectif (sélection participative) s'est ainsi étendu à plusieurs espèces sur tout le territoire. Les pays du Sud nous avaient précédés pour pallier les insuffisances de la révolution verte; en Europe, les Portugais furent les premiers à s'organiser pour la sauvegarde de leur patrimoine variétal et leur tradition. En France, notre équipe a accompagné plusieurs associations membres du Réseau semences paysannes. Chaque expérience commence par l'exploration de variétés diversifiées et le plus souvent "différentes " de celles du marché pour explorer les capacités d'adaptation et le potentiel de qualité. Le rôle des chercheurs est alors de "fouiller" dans les banques de graines aux quatre coins du monde pour retrouver de la diversité génétique. Les échantillons envoyés par les centres de ressources génétiques sont souvent très petits et nécessitent un travail de multiplication qui s'organise différemment selon le mode de reproduction de l'espèce (allogamie/autogamie), et la structure ou l'effectif du groupe d'agriculteurs demandeurs. Cette multiplication peut se faire chez les agriculteurs directement. La compétence de I'animateur d'association (souvent un ingénieur) est alors nécessaire pour coordonner les actions (figure 4) des points de vue organisationnel et technique (qui a quoi, quelles sont les règles à respecter pour la multiplication selon la botanique de l'espèce, quelles observations...). Dans certains cas, la multiplication nécessite une collaboration avec la recherche où les informations sur la génétique, la biologie et le cycle de culture de la plante sont plus facilement accessibles (expérience technique et bibliographie quand les savoir-faire ont disparu ou sont spécifiques des ressources collectées). Les premières évaluations au champ (même en toutes petites parcelles) nécessitent déjà des quantités de graines qui demandent deux ou trois ans de travail préalable. Le processus de sélection pour adapter les variétés aux nouvelles conditions pratiques et aux critères de qualité demande alors une collaboration forte entre chercheurs, animateurs d'associations et agriculteurs et associe leurs savoir-faire (traditionnel et/ou innovant). Le travail de recherche est souvent justifié par la nécessité de créer des savoir-faire qui ont disparu des campagnes avec l'industrialisation de I'agriculture. Les nouveaux savoir-faire sont le fruit de la collaboration entre chercheurs et agriculteurs, les uns apportant des éléments théoriques que les seconds enrichissent (et vice versa), fonction de la créativité et de l'expérience des uns et des autres (figure 5). 


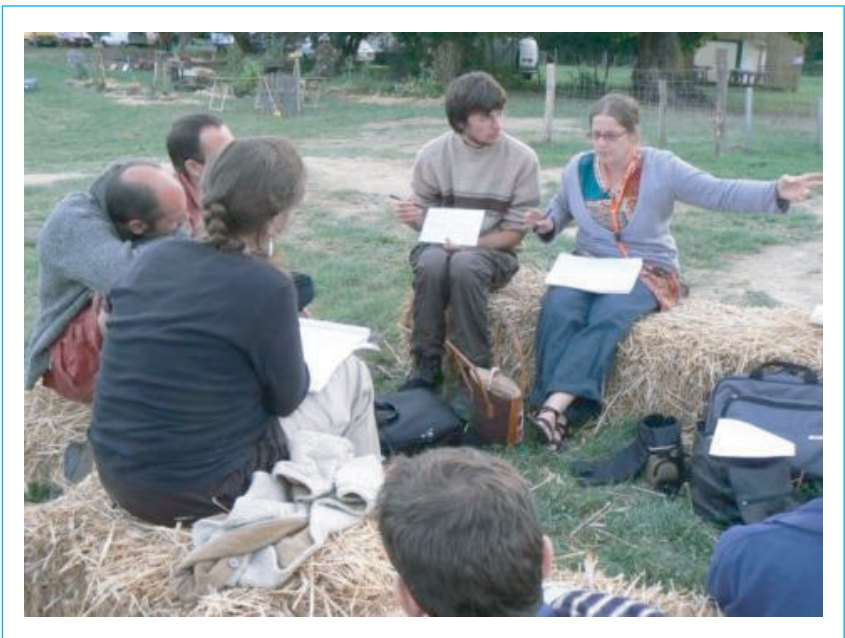

Figure 4 - Rencontre autour d'un projet de recherche : mise au point des schémas d'expérimentation

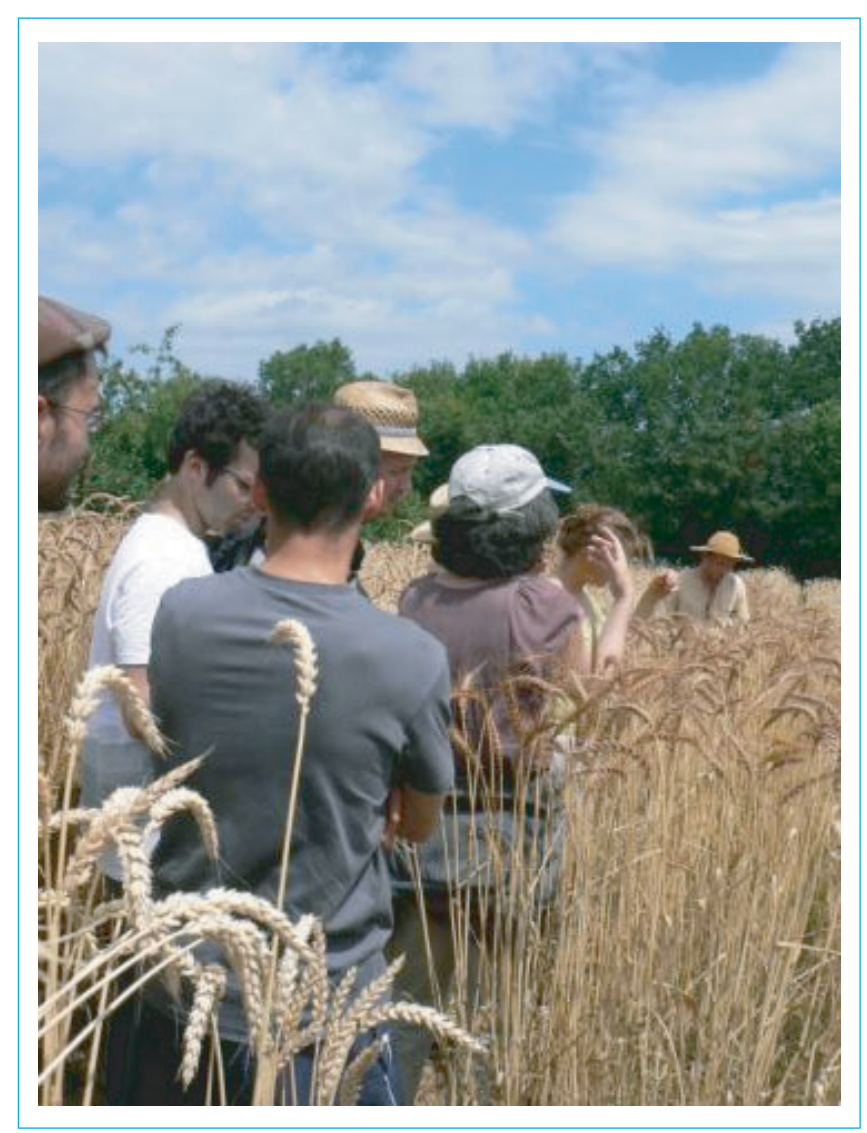

Figure 5 - Visite à la ferme des essais de descendances de croisement biparentaux de populations de blé

Diversité oblige, les agriculteurs bio ont commencé à solliciter les recherches pour explorer des espèces peu cultivées, laissées de côté, comme la cameline pour les crucifères (huile), le carthame, les fourragères...
Les réseaux se multiplient et grandissent. Le vocabulaire évolue: on parle de moins en moins de variétés anciennes mais de variétés paysannes (référence aux agriculteurs qui les travaillent) ou de variétés populations (référence à leur niveau de diversité, par opposition aux variétés commerciales stables et homogènes, qui sont des lignées, des hybrides F1 ou des clones). De nouveaux types de variétés sont particulièrement créés pour la sélection paysanne, comme les mélanges dynamiques ou les CCP (Composites Cross Populations) (voir encadré 2).

Pour chacune des espèces travaillées, des questions particulières, dépendantes du contexte sont travaillées par des groupes multi-acteurs et transdisciplinaires comme illustré dans le tableau 1.

La communauté des agriculteurs qui se réapproprient les semences pour développer des variétés adaptées à leur conditions d'agriculture biologique est maintenant assez importante et les récoltes de variétés paysannes sont vendues et transformées; ce changement d'échelle renouvelle les questions (processus itératif) comme l'interaction entre les variétés et les différents environnements où elles sont cultivées, leurs capacités d'adaptation à ces environnements, aux pratiques des agriculteurs, et cela jusqu'au produit fini. Les conséquences des différents niveaux de diversité des variétés (notamment des variétés créées par les agriculteurs), du champ à l'assiette, sont autant de questions que se posent les praticiens dans leur volonté d'approfondissement et de connaissance de leurs outils. Notre rôle de chercheur est d'accompagner tous les acteurs d'un point de vue méthodologique, de participer à la recherche de ces réponses, en relation avec les agriculteurs, les animateurs d'associations de producteurs, et de plus en plus les nouveaux acteurs du système alimentaire qui rejoignent légitimement ce travail de recherche participative.

\subsection{De la sélection à la recherche participative, de la variété aux systèmes alimentaires}

\subsection{1 Élargissement du champ des recherches}

Notre travail de recherche, dont le point de départ est l'étude comportementale des ressources génétiques et variétés paysannes, s'est élargi au cours des années avec les nouvelles questions des praticiens concernant l'ensemble du processus de production du sol à l'assiette. Nos compétences se trouvent vite limitées face à des questions comme " comment nos variétés interagissent-elles dans le sol avec les mycorhizes, les mycorhizes étant des champignons symbiotiques des racines facilitant l'alimentation minérale de la plante" ? Nous devons donc élargir nos connaissances scientifiques et surtout nous entourer de collègues, scientifiques (chercheurs, ingénieurs ou techniciens) et praticiens spécialisés dans les différents domaines à approfondir.

Cet élargissement des thématiques de recherche autour des variétés pour l'agriculture biologique induit une évolution du métier de chercheur: la sélection participative devient une recherche participative transdisciplinaire. La génétique appliquée à l'amélioration des plantes s'enrichit donc des disciplines nécessaires pour aborder le système alimentaire du champ à l'assiette. Nous établissons progressivement une transdisciplinarité stimulante, passant de l'étude de la plante à l'étude des systèmes alimentaires. Un point fort pour pratiquer la transdisciplinarité est d'associer une discipline spécifique (génétique/amélioration des plantes, entre autres) à une formation " généraliste " d'ingénieur agronome que nous avons entretenue par un contact régulier avec la réalité du terrain. Celle-ci assure une vision globale et un pragmatisme préconisés par les pionniers de la bio, tout en approfondissant une "dominante " (et non pas la spécialité) "diversité cultivée ". 


\section{Tableau 1 - Recherche participative pour cinq espèces cultivées} (brocoli, tomate, blé, maïs et sarrasin)

\begin{tabular}{|c|c|c|c|c|c|}
\hline Espèce cultivée & Objectif & Contexte & Problématique & Méthodes & Partenaires et moyens \\
\hline & $\begin{array}{l}\text { Créer des variétés adaptées } \\
\text { à la bio, au goût remarqua- } \\
\text { ble dans un processus } \\
\text { associant sélections partici- } \\
\text { pative et professionnelle }\end{array}$ & $\begin{array}{l}\text { La forme connue du brocoli } \\
\text { est une création récente } \\
\text { dont la majorité des variétés } \\
\text { comporte des biotechnolo- } \\
\text { gies incompatibles avec la } \\
\text { bio } \\
\text { Lieu : Ouest et Sud de la } \\
\text { France }\end{array}$ & $\begin{array}{l}\text { Comment, à partir de } \\
\text { variétés locales tradi- } \\
\text { tionnelles conservées } \\
\text { dans des centres de res- } \\
\text { sources génétiques, } \\
\text { associer les goûts variés } \\
\text { et des formes/couleurs } \\
\text { originales peu connus } \\
\text { des consommateurs et } \\
\text { favoriser le caractère } \\
\text { adaptatif des variétés? }\end{array}$ & $\begin{array}{l}\text { Après évaluation de res- } \\
\text { sources génétiques, des } \\
\text { nouvelles populations } \\
\text { sont créées par croise- } \\
\text { ments (sélectionneurs } \\
\text { privés) puis évaluées } \\
\text { (sélectionneurs et agri- } \\
\text { culteurs) sur des critè- } \\
\text { res de robustesse } \\
\text { agronomique et de qua- } \\
\text { lité organoleptique }\end{array}$ & $\begin{array}{l}\text { Partenaires : sélection- } \\
\text { neurs privés, plateforme } \\
\text { collective d'agriculteurs } \\
\text { et agriculteurs indivi- } \\
\text { duels, recherche } \\
\text { Moyens : projet } \\
\text { européen SOLIBAM } \\
\text { (2010-2014) qui se pour- } \\
\text { suit dans un second } \\
\text { DIVERSIFOOD } \\
\text { (2015-2019) }\end{array}$ \\
\hline & $\begin{array}{l}\text { Proposer et évaluer } \\
\text { des méthodes de sélection } \\
\text { à la ferme en associant la } \\
\text { sélection professionnelle } \\
\text { pour créer des variétés } \\
\text { diversifiées et avec un bon } \\
\text { goût }\end{array}$ & $\begin{array}{l}\text { La diversité en tomate a } \\
\text { commencé son entrée sur le } \\
\text { marché mais on observe } \\
\text { une confusion avec des } \\
\text { variétés hybrides qui ont } \\
\text { repris le nom de variétés } \\
\text { anciennes et quelques uns } \\
\text { des traits morphologiques } \\
\text { typiques sans en garder la } \\
\text { typicité sensorielle } \\
\text { Lieu : Sud de la France, } \\
\text { collaboration avec Italie }\end{array}$ & $\begin{array}{l}\text { Est-il possible de sélec- } \\
\text { tionner pour des critè- } \\
\text { res qualitatifs et de } \\
\text { maintenir une diversité } \\
\text { nécessaire au caractère } \\
\text { adaptatif? }\end{array}$ & $\begin{array}{l}\text { Des croisements de } \\
\text { parents des deux grou- } \\
\text { pes de populations, } \\
\text { "Marmande " et } \\
\text { "Cœur de bœuf " ont } \\
\text { été réalisés pour étudier } \\
\text { les diversités de formes } \\
\text { et de qualités organo- } \\
\text { leptiques dans les des- } \\
\text { cendances }\end{array}$ & $\begin{array}{l}\text { Partenaires : sélection- } \\
\text { neurs privés, agricul- } \\
\text { teurs individuels, } \\
\text { recherche } \\
\text { Moyens : projet } \\
\text { européen SOLIBAM } \\
\text { (2010-2014) qui se } \\
\text { poursuit dans un second } \\
\text { DIVERSIFOOD } \\
\text { (2015-2019) }\end{array}$ \\
\hline Blé & $\begin{array}{l}\text { Évaluer des stratégies de } \\
\text { création de diversité sur } \\
\text { des critères adaptatifs et de } \\
\text { qualité des farines dont } \\
\text { celle nécessaire pour la } \\
\text { boulange traditionnelle au } \\
\text { levain naturel pour sélec- } \\
\text { tionner des populations de } \\
\text { blés à la ferme }\end{array}$ & $\begin{array}{l}\text { Il est indispensable de } \\
\text { redonner de la diversité } \\
\text { intrinsèque aux variétés de } \\
\text { blés pour renforcer leur } \\
\text { robustesse. Les variétés tra- } \\
\text { ditionnelles maintenues en } \\
\text { " banque de gènes " ont } \\
\text { perdu leur diversité initiale } \\
\text { et les variétés modernes } \\
\text { sont des lignées pures } \\
\text { Lieu : toute la France }\end{array}$ & $\begin{array}{l}\text { Plusieurs stratégies ont } \\
\text { été initiées par les cher- } \\
\text { cheurs et paysans. } \\
\text { Quels sont les atouts et } \\
\text { limites des méthodes } \\
\text { utilisées par les } \\
\text { agriculteurs? }\end{array}$ & $\begin{array}{l}\text { Observation fine des } \\
\text { évolutions de structu- } \\
\text { res variétales, et des } \\
\text { populations à la base de } \\
\text { leur composition (popu- } \\
\text { lation dynamique et } \\
\text { CCP, composite cross } \\
\text { population) }\end{array}$ & $\begin{array}{l}\text { Partenaires : agricul- } \\
\text { teurs, animateurs d'asso- } \\
\text { ciations, recherche } \\
\text { Moyens : projets } \\
\text { européens SOLIBAM } \\
\text { (2010-2014), COBRA } \\
\text { (2013-2016) et DIVERSI- } \\
\text { FOOD (2015-2019); } \\
\text { projets locaux } \\
\text { (fondations privées) }\end{array}$ \\
\hline & $\begin{array}{l}\text { Comprendre l'évolution } \\
\text { des populations et recher- } \\
\text { che de critères d'évaluation } \\
\text { de la qualité facile à mesu- } \\
\text { rer à la ferme et par les utili- } \\
\text { sateurs pour l'alimentation } \\
\text { humaine et pour le fourrage }\end{array}$ & $\begin{array}{l}\text { Les populations tradition- } \\
\text { nelles de maïs cultivées } \\
\text { dans le sud ouest de la } \\
\text { France pour I'alimentation } \\
\text { humaine ont été très rédui- } \\
\text { tes depuis I'expansion de } \\
\text { la culture des hybrides F1. } \\
\text { Les agriculteurs bio et } \\
\text { conventionnels évaluent } \\
\text { des populations variées et } \\
\text { en créent des nouvelles } \\
\text { Lieu : grand ouest de la } \\
\text { France de la Bretagne } \\
\text { au Pays basque }\end{array}$ & $\begin{array}{l}\text { Quelles stratégies } \\
\text { de sélection pour amé- } \\
\text { liorer les populations } \\
\text { sur leur robustesse et } \\
\text { la qualité des grains, } \\
\text { renouer avec la culture } \\
\text { culinaire locale et créer } \\
\text { des nouvelles formes } \\
\text { de valorisation }\end{array}$ & $\begin{array}{l}\text { Mettre en œuvre } \\
\text { différents protocoles } \\
\text { de sélection sur des } \\
\text { structures collectives } \\
\text { et des fermes avec des } \\
\text { approches analytiques } \\
\text { pour la qualité }\end{array}$ & $\begin{array}{l}\text { Partenaires : agriculteurs } \\
\text { (collaborations avec pay- } \\
\text { sans du Brésil et du Mexi- } \\
\text { que) pour importer des } \\
\text { stratégies de sélection } \\
\text { paysannes et des popu- } \\
\text { lations diversifiées } \\
\text { Moyens : projets } \\
\text { régions, notamment } \\
\text { I'Aquitaine (figure 6), la } \\
\text { Bretagne et Pays de } \\
\text { Loire, projets européens } \\
\text { (SOLIBAM 2010-2014; } \\
\text { DIVERSIFOOD } \\
\text { 2015-2019) }\end{array}$ \\
\hline & $\begin{array}{l}\text { Rechercher des sources } \\
\text { de diversité notamment } \\
\text { dans les pays du monde où } \\
\text { l'espèce a été domestiquée } \\
\text { afin de créer des popula- } \\
\text { tions plus robustes et diver- } \\
\text { sifiées pour ses usages. } \\
\text { L'espèce sous-utilisée est } \\
\text { très recherchée aujourd'hui } \\
\text { pour ses intérêts agronomi- } \\
\text { ques, son caractère sans } \\
\text { gluten et sa richesse } \\
\text { nutritionnelle }\end{array}$ & $\begin{array}{l}\text { Le sarrasin souffre d'un } \\
\text { manque de variétés sur le } \\
\text { marché. Il a été abandonné } \\
\text { par la recherche publique et } \\
\text { les sélectionneurs privés } \\
\text { Lieu : Bretagne en réseau } \\
\text { avec des associations } \\
\text { nationales }\end{array}$ & $\begin{array}{l}\text { Comment refaire vivre } \\
\text { la diversité de cette } \\
\text { espèce dont de très } \\
\text { nombreux échantillons } \\
(5000) \text { dorment dans } \\
\text { les conservatoires de } \\
\text { ressources génétiques } \\
\text { et quelle stratégie de } \\
\text { sélection adopter à la } \\
\text { ferme pour augmenter } \\
\text { sa robustesse? }\end{array}$ & $\begin{array}{l}\text { Créer un réseau de par- } \\
\text { rainage pour multiplier } \\
\text { en isolement de nom- } \\
\text { breux petits échan- } \\
\text { tillons (65), évaluer la } \\
\text { diversité au sein des } \\
\text { ressources génétiques } \\
\text { et initier un programme } \\
\text { de sélection à la ferme }\end{array}$ & $\begin{array}{l}\text { Partenaires : paysans, } \\
\text { apiculteurs, lycée agri- } \\
\text { cole, meuniers, crêpiers } \\
\text { Moyens : Fondation de } \\
\text { France et projet euro- } \\
\text { péen (DIVERSIFOOD } \\
\text { 2015-2019) }\end{array}$ \\
\hline
\end{tabular}

L'évolution de notre métier a été stimulée à travers quelques projets coordonnés par notre équipe et couvrant le système alimentaire dans son ensemble. Le contenu des trois programmes européens successifs (dans trois programmes cadres successifs de la Commission européenne, FP6, FP7 et H2020), coordonnés par notre équipe, illustre ainsi la progression collective vers la transdisciplinarité, retentissant également sur le métier de chacun.
Le projet de recherche européen Farm Seed Opportunities (2007-2010) avait pour objectif de faire un état des lieux des variétés "non conventionnelles" (non inscrites au catalogue officiel des variétés) utilisées en Europe, de les décrire pour aider à leur prise en compte par les institutions européennes en charge de la réglementation. Ce projet comportait une étude de l'évolution des variétés populations (de leur plasticité) dans des conditions 


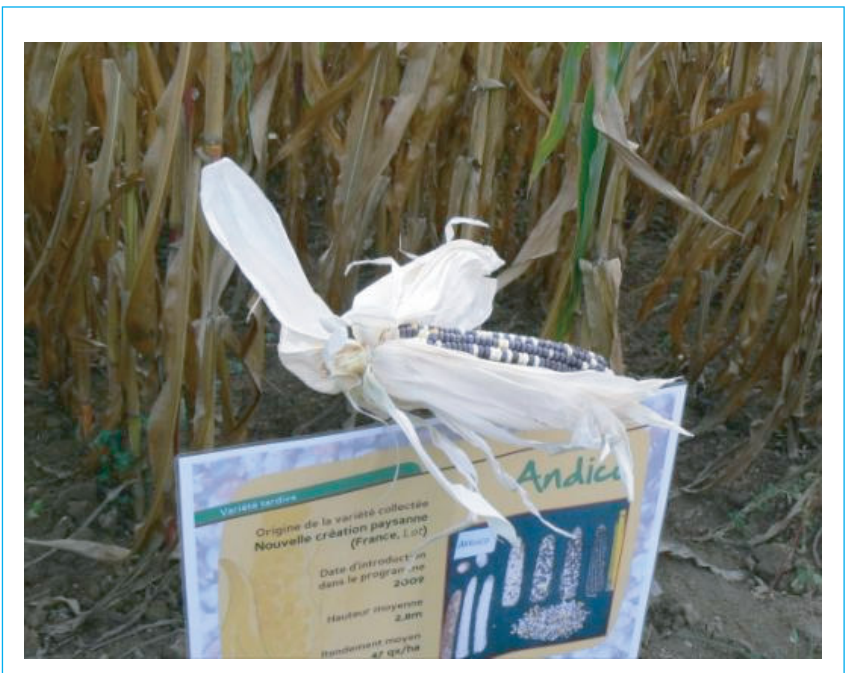

Figure 6 - Essai à la ferme de nouvelles populations de maïs. Les évaluations sont organisées collectivement sur une ferme désignée " plateforme " où des rencontres sont organisées tous les ans depuis 2002 par AgroBioPérigord en Aquitaine

d'agriculture biologique variées. II s'agissait d'apporter des éléments scientifiques sur leur potentiel d'adaptation. Les variétés étudiées en France, Pays-Bas et Italie étaient des variétés populations déjà cultivées par des agriculteurs. Nous organisions une recherche à la ferme orientée par les objectifs des agriculteurs (avoir des éléments tangibles sur la plasticité des populations). Sur le plan des disciplines, étaient associés des agronomes, des généticiens, des pathologistes et des sociologues. Ce projet a impliqué plusieurs chercheurs et agriculteurs mais n'était pas un projet de recherche participative. En effet, l'objet n'était pas le processus de production lui-même, mais une collaboration en vue de l'obtention de données démontrant la grande plasticité et le potentiel d'adaptation des populations pour l'agriculture biologique, afin de proposer des aménagements à la réglementation semencière en vigueur. Notre équipe, outre la coordination du projet qui a nourri le passage de la multidisciplinarité à la transdisciplinarité, s'est chargée de la coordination des essais et de leur suivi technique dans plusieurs pays. L'ingénieur a été le lien entre les chercheurs, les techniciens et agriculteurs dans la mise en œuvre des protocoles de semis et d'observation afin de trouver des compromis entre la vision théorique des essais agronomiques et une application à la ferme qui soit suffisante pour obtenir une bonne qualité des données et une implantation à la ferme qui soit supportable pour les agriculteurs.

- SOLIBAM (Strategies for Organic and Low input Integrated Breeding and Management, 2010-2014) a succédé à Farm Seed Opportunities. II avait pour objectif d'étudier différentes stratégies de sélection en relation avec les pratiques culturales pour les agricultures biologiques et à faibles intrants dans une collaboration entre 23 partenaires de 12 pays. Il était fondé sur une hypothèse : la " diversité " déployée à tous niveaux, du champ à l'assiette, améliore la performance, la durabilité, la stabilité des systèmes de cultures et la qualité des produits. Les partenaires ont développé des stratégies de sélection sur le blé, l'orge, le maïs, la féverole, le haricot, la tomate et le brocoli, intégrées à des expérimentations agronomiques, pour créer ou stimuler la diversité au sein des peuplements végétaux, et mesurer son impact sur la durabilité des systèmes de production. II s'appuyait parfois sur des projets locaux, comme le projet PaysBlé dans l'Ouest de la France, sur la thématique du blé, suivi par notre équipe. Ce projet local avait pour objectif d'étudier les impacts, du champ jusqu'à l'assiette, de différents types variétaux de blé tendre, afin d'identifier des éléments stratégiques du processus aidant les agriculteurs et boulangers à améliorer leur production en matière de sélection variétale et de pratiques à développer. Ce projet est né suite à des questions d'agriculteurs et de boulangers bio au sein du Réseau semences paysannes. Pour balayer le sujet "du grain au pain", nous avons travaillé avec des chercheurs et des praticiens des disciplines impliquées tout au long du processus de transformation: agronomie, meunerie, boulange, analyse sensorielle. Certaines de ces actions ont été développées en collaboration très étroite entre chercheurs et praticiens, et aussi avec les consommateurs lors des dégustations de pain, confrontant ainsi nos travaux de recherche aux aspirations des citoyens. Avec le projet PaysBlé, nous avons évalué les impacts et interactions de plusieurs facteurs (variété environnement et conduite culturale, technique de panification) sur les performances (quantitatives et qualitatives) de l'ensemble du processus. Par exemple, d'un point de vue agronomique, nous avons observé que les variétés de blé plus diversifiées génétiquement avaient un rendement plus stable à travers les lieux et les années. Sur le plan de la qualité organoleptique (figure 7), nous avons mis en lumière que l'environnement de culture influençait plutôt la texture du pain alors que la variété avait un impact sur le goût [73]. Pour la mise au point des protocoles et les analyses couvrant la totalité du processus, des discussions précises et techniques ont eu lieu entre les référents des différentes disciplines. Cette collaboration, dans laquelle le rôle de l'ingénieur est central car il permet une traduction entre toutes les disciplines de par sa vision globale, a été une clé pour la réussite de la transdisciplinarité du projet. En outre, l'approfondissement méthodologique, ainsi qu'une prise de recul sur l'organisation des acteurs, ont fait l'objet d'une thèse de doctorat [74].

Avec Diversifood (Embedding crop diversity and networking for local high quality food systems, nouveau projet de recherche européen qui a commencé en 2015), la problématique s'élargit à de nombreuses espèces sous-utilisées pour enrichir les paysages agricoles et la diversité des systèmes de culture, et donc leur résilience.

Grâce à l'expérience que nous avons de la bio paysanne par le contact régulier avec des praticiens, souvent chercheurs/expérimentateurs sur leur ferme, notre métier d'ingénieurs-chercheurs du vivant s'enrichit au fil du temps. Cette évolution se traduit dans les différents projets. Cette expérience (de type jurisprudentielle ou expérientielle) se transmet par la formation de nombreux stagiaires de tous niveaux, de doctorants et d'ingénieurs en contrat sur les projets.

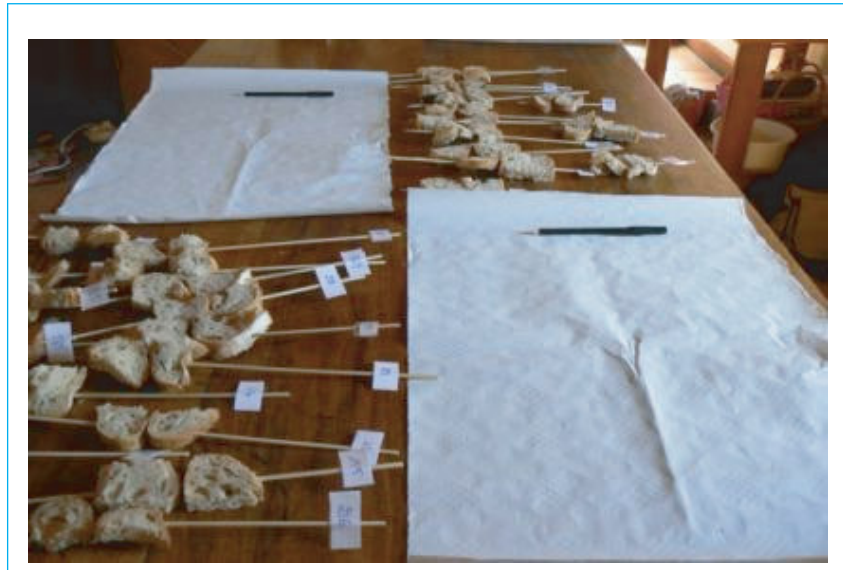

Figure 7 - Échantillons de pain pour évaluation de la qualité organoleptique en utilisant une méthode de Napping [74] ne nécessitant pas d'entraînement et à la portée de tous 


\subsubsection{Méthode de travail pour une vision globale}

Retrouver des variétés diversifiées et évolutives, sélectionnées et multipliées à la ferme, ne soulève pas les mêmes questions d'une espèce à l'autre. Les repositionner dans un système alimentaire révèle des goulots d'étranglement qui sont d'ordres différents et font appel à des compétences variées. Le tableau 2 recense, pour cinq espèces, la problématique générale dans laquelle s'inscrit le redéploiement de la diversité de cette espèce : un règlement sanitaire pour le haricot, les facteurs de qualité pour le blé, la création de nouveaux débouchés pour le maïs, le manque de diversité et un marché tourné vers l'importation pour le sarrasin, et la réinsertion de l'arbre dans ou autour de champs cultivés avec le châtaignier.

Avec ces exemples, nous illustrons concrètement comment la recherche globale, encouragée pour la bio, se décompose en quatre types d'approche (définis dans les deux axes du carré de Bawden, objectif/subjectif, holisme/réductionnisme, voir paragraphe 2.3) selon les goulots d'étranglement identifiés dans la problématique. L'approche globale est de savoir " se promener " dans ces carrés et relativiser les activités associées à sa discipline dans ces quatre types de champ. Ce cheminement d'un carré à l'autre aide à clarifier le champ de validité des hypothèses de chacun des champs disciplinaires et les confronte à la réalité des systèmes alimentaires dans ses composantes biologique, socio-économique et culturelle.

Ce passage d'un champ de référence à l'autre dans le carré de Bawden est un processus itératif, comme le décrit Ton Baars [35]. Chaque passage introduit des questions nouvelles toujours aussi concrètes et non pas une application théorique. Cependant, nous abordons néanmoins de grandes questions conceptuelles, telle que la santé des plantes, la qualité des aliments qui s'enrichissent de nos études de cas et prennent une forme de généricité pour conforter les hypothèses des pionniers de la bio.

\subsubsection{Au-delà de la technique, les champs politiques et sociaux}

Le troisième carré de Bawden, généralement peu investi par les sciences biotechniques, est le lieu de confrontation avec les réalités sociales, économiques et réglementaires.

Une des réalités sociales au cœur de nos activités de sélection avec les paysans est la création ou le renforcement du système semencier informel. Dans certains pays du Sud, il existe encore un système semencier local mais dans les pays d'Europe, il est en cours de renaissance avec l'établissement de réseaux de semences paysannes [75] : en France, le Réseau semences paysannes (RSP), en Espagne, la Red de Semilla (RdS) et en Italie, la Rete Semi Rurali. Leurs caractéristiques et leurs organisations sont similaires : associer différents acteurs de la société civile concernés par la biodiversité cultivée (associations, syndicats agricoles, institutions...). Ces organisations ont souvent été créées au début des années 2000, avec une expansion rapide, mesurée par le nombre d'associations adhérentes et par le nombre d'actions menées (projets de recherche en partenariat, foire à la biodiversité, publications, formations...). Elles ont un fort lien avec la société civile, inquiète de la qualité de l'alimentation et de la perte de la diversité. Ces initiatives sont aussi fédérées au niveau européen par la coordination Let's Liberate Diversity et ont des activités à l'échelle internationale. Les membres de ces associations sont acteurs de la

\section{Tableau 2 - Recherche participative pour cinq espèces (haricot, blé, maïs, sarrasin et châtaigner) : organisation des actions de recherche selon les critères définis par les axes du carré de Bawden}

\begin{tabular}{|c|c|c|c|c|c|}
\hline Espèces & $\begin{array}{l}\text { Problématique } \\
\text { générale }\end{array}$ & $\begin{array}{c}\text { Carré } 1 \text { : travail } \\
\text { analytique pour des } \\
\text { études approfondies } \\
\text { de mécanismes du vivant }\end{array}$ & $\begin{array}{l}\text { Carré } 2 \text { : analyse } \\
\text { systémique dans } \\
\text { les dimensions } \\
\text { agronomiques } \\
\text { et écologiques }\end{array}$ & $\begin{array}{l}\text { Carré } 3 \text { : construction } \\
\text { socio-économique, } \\
\text { débats éthiques } \\
\text { et culturels }\end{array}$ & $\begin{array}{c}\text { Carré } 4 \text { : appropriation } \\
\text { individuelle, } \\
\text { partage d'expériences }\end{array}$ \\
\hline Haricot & $\begin{array}{l}\text { Diversité et santé des } \\
\text { plantes dans un cadre } \\
\text { réglementaire inadapté } \\
\text { et conflictuel }\end{array}$ & $\begin{array}{l}\text { Observation des mycorhizes } \\
\text { présents sur les racines de } \\
\text { populations de haricots. Ana- } \\
\text { lyse génomique de la diver- } \\
\text { sité microbienne sur les } \\
\text { semences. Détermination des } \\
\text { maladies présentes en culture }\end{array}$ & $\begin{array}{l}\text { Étude de la relation entre } \\
\text { la diversité génétique des } \\
\text { plantes, les pratiques et } \\
\text { celles de microorganis- } \\
\text { mes présents sur les } \\
\text { semences }\end{array}$ & $\begin{array}{l}\text { Les résultats des " carrés " } 1 \\
\text { et } 2 \text { constituent le socle de } \\
\text { débats réglementaires et de } \\
\text { propositions d'évolution } \\
\text { pour prendre en compte } \\
\text { les principes de la bio }\end{array}$ & $\begin{array}{l}\text { Recueil d'expériences et } \\
\text { expérimentation chez les } \\
\text { praticiens (paysans arti- } \\
\text { sans-semenciers) avec des } \\
\text { pratiques individualisées } \\
\text { et innovantes }\end{array}$ \\
\hline Blé & $\begin{array}{l}\text { Qualité : comment agir } \\
\text { du grain au pain pour } \\
\text { proposer un pain } \\
\text { traditionnel de qualité }\end{array}$ & $\begin{array}{l}\text { Adaptation de méthodes de } \\
\text { mesure de la qualité, suivi } \\
\text { des paramètres du rende- } \\
\text { ment, suivi de la vie du sol. } \\
\text { Analyse génétique de la } \\
\text { diversité }\end{array}$ & $\begin{array}{l}\text { Étude des relations } \\
\text { et interactions entre les } \\
\text { éléments de l'agro- } \\
\text { écosystème du sol } \\
\text { au pain }\end{array}$ & $\begin{array}{l}\text { Validation de l'intérêt de la } \\
\text { diversité cultivée auprès des } \\
\text { autres acteurs locaux et poli- } \\
\text { tiques (du local à l'Europe), } \\
\text { révision de la réglementa- } \\
\text { tion sur les semences en } \\
\text { Europe }\end{array}$ & $\begin{array}{l}\text { Intégration des outils } \\
\text { d'aide à la décision, } \\
\text { repositionnement des } \\
\text { questions par les cher- } \\
\text { cheurs, les paysans et } \\
\text { paysans-boulangers }\end{array}$ \\
\hline Maïs & $\begin{array}{l}\text { Diversifier : élargir le } \\
\text { champ des acteurs }\end{array}$ & $\begin{array}{l}\text { Adaptation de méthodes de } \\
\text { mesure de la qualité. Mesure } \\
\text { des paramètres du rende- } \\
\text { ment. Étude la qualité des } \\
\text { farines en fonction des recet- } \\
\text { tes }\end{array}$ & $\begin{array}{l}\text { Recherche de critères phé- } \\
\text { notypiques et physiologi- } \\
\text { ques liés à la qualité } \\
\text { nutritionnelle en fonction } \\
\text { des pratiques et de l'envi- } \\
\text { ronnement }\end{array}$ & $\begin{array}{l}\text { Élargissement du réseau } \\
\text { d'acteurs pour valoriser la } \\
\text { diversité des maïs et créa- } \\
\text { tion de nouvelles recettes en } \\
\text { lien avec la diversité collec- } \\
\text { tée dans le monde et créée à } \\
\text { la ferme }\end{array}$ & $\begin{array}{l}\text { Appropriation de la diver- } \\
\text { sité par les profession- } \\
\text { nel/artistes de la cuisines. } \\
\text { Créativité des paysans } \\
\text { pour adapter le maïs à } \\
\text { leurs pratiques }\end{array}$ \\
\hline Sarrasin & $\begin{array}{l}\text { Diversifier dans une } \\
\text { filière très réduite }\end{array}$ & $\begin{array}{l}\text { Étude de la diversité généti- } \\
\text { que (marqueurs moléculaires) } \\
\text { et phénotypique }\end{array}$ & $\begin{array}{l}\text { Relation entre diversité } \\
\text { génétique, comportement } \\
\text { des pollinisateurs, prati- } \\
\text { ques culturales et environ- } \\
\text { nement }\end{array}$ & $\begin{array}{l}\text { Sensibilisation de la filière à } \\
\text { I'intérêt de la diversité pour } \\
\text { relocaliser la production. } \\
\text { Implication des } \\
\text { consommateurs pour la } \\
\text { renaissance d'une produc- } \\
\text { tion traditionnelle }\end{array}$ & $\begin{array}{l}\text { Intégration des outils } \\
\text { d'aide à la décision par les } \\
\text { paysans apiculteurs, crê- } \\
\text { piers... pour optimiser les } \\
\text { performances de leur pro- } \\
\text { duction }\end{array}$ \\
\hline Châtaignier & $\begin{array}{l}\text { Diversifier en } \\
\text { réintégrant l'arbre } \\
\text { dans le processus } \\
\text { productif }\end{array}$ & $\begin{array}{l}\text { Analyse de la diversité } \\
\text { "rescapée " dans les zones } \\
\text { traditionnelles. Adaptation de } \\
\text { méthodes d'analyses de qua- } \\
\text { lité }\end{array}$ & $\begin{array}{l}\text { Évaluation des relations } \\
\text { entre techniques de pro- } \\
\text { duction (haies, agrofores- } \\
\text { terie) et produits } \\
\text { valorisables }\end{array}$ & $\begin{array}{l}\text { Déterminer des scénarios de } \\
\text { révalorisation de l'espèce } \\
\text { avec l'ensemble de la chaîne } \\
\text { alimentaire }\end{array}$ & $\begin{array}{l}\text { Stimuler l'innovation } \\
\text { locale pour la mise en } \\
\text { valeur de l'arbre (à la } \\
\text { ferme, la transformation } \\
\text { et l'artisanat) }\end{array}$ \\
\hline
\end{tabular}


recherche participative avec qui nous travaillons au quotidien. Nous faisons même partie de certaines de ces associations et avons participé à leur création. Elles sont un maillon indispensable dans un cadre réglementaire où la semence paysanne ne peut pas être commercialisée. De leur organisation et leur animation dépendent aussi une partie de l'efficacité du travail collectif. Elles représentent les acteurs de terrain (paysans, boulangers, consommateurs, jardiniers...) et sont souvent partenaires officiels des projets de recherche financés par les régions, les fondations et même la Commission européenne.

Sur le plan économique, nous sommes directement en lien avec les circuits courts, par les agriculteurs avec qui nous travaillons, mais aussi d'un point de vue personnel, et cela nous permet par exemple de mobiliser de temps à autre les consommateurs pour des séances de dégustation. Cependant, l'émergence des semences paysannes aurait avantage à avoir une visibilité plus grande dans les circuits courts et locaux, et même plus large avec le réseau Biocoop. Des réflexions sont en cours dans les associations paysannes pour essayer d'imaginer un système de repérage des produits issus de semences paysannes dans toutes les formes de distribution. Les implications économiques de l'investissement en semences paysannes dans l'activité des fermes commencent aussi à devenir un sujet d'étude demandé par les paysans et qui interroge de nombreux observateurs du phénomène des semences paysannes.

Le cadre réglementaire est celui qui a mobilisé le plus d'effort depuis le début des projets de sélection participative. Le défi était de redonner une place légale à la semence paysanne et de proposer des aménagements pour les besoins de l'agriculture biologique en général [64]. Les trois programmes européens Farm Seed Opportunities, SOLIBAM et maintenant Diversifood, ont été concus avec une large interaction avec les législateurs européens (plusieurs séminaires de travail à la Commission européenne pour partager les résultats de nos recherches sur le terrain) pour concevoir la prochaine réglementation semences en adéquation avec les évolutions que nous accompagnons. Le Parlement n'a pas voté la proposition de loi issue de ce travail de négociation, mais le secteur semencier professionnel, représenté par le GNIS et I'UFS (Union française des semenciers) en France, a reconnu qu'elle représentait une avancée pour tous les opérateurs: "Souhaitant depuis longtemps que la réglementation soit adaptée pour des variétés anciennes ou des variétés pour des marchés amateurs ou locaux, pour le secteur des semences, la Commission avait réalisé un travail considérable pour actualiser, simplifier et mettre en cohérence les douze directives existantes sur la production et la commercialisation des semences [76] ". Notre groupe de recherche, associé aux acteurs paysans et professionnels, va continuer à œuvrer pour un contexte réglementaire en adéquation avec la réalité des campagnes européennes.

Un projet français soutenu par le ministère de l'Écologie, ReSoRIV [77], avait la même ambition au plan national, en questionnant en outre les décideurs de la recherche pour mieux intégrer la recherche participative dans les institutions. Se pose en effet la question des moyens humains pour soutenir et renforcer les forces de recherche qui sont limitées actuellement à des initiatives qui, bien que connectées entre elles et à d'autres initiatives européennes, restent encore individuelles. Des étudiants en thèse sont aussi formés dans nos laboratoires, auront-ils des perspectives de carrière de chercheurs ? Comme les recrutements sont en nombre très limité à I'INRA, seules des ambitions politiques peuvent soutenir des voies scientifiques nouvelles en réorientant la politique des ressources humaines de l'institut, sinon les nouvelles compétences trouveront un avenir hors de la recherche publique.

En matière de recommandations politiques, les chercheurs du projet SOLIBAM encourageaient aussi à réviser l'évaluation des productions des chercheurs pour que l'engagement en recherche participative ne soit pas regardé seulement comme une démarche militante.
Notre travail d'ingénieur-chercheur a évolué au cours des quinze années d'expérience de recherche autour de la biodiversité cultivée. Cet aspect, toujours au cœur de notre métier est aussi facteur d'élargissement du champ d'action depuis la sélection participative jusqu'aux systèmes alimentaires en intégrant les différents acteurs de ces systèmes. Notre recherche se qualifie de "participative", " multi-acteurs " et " transdisciplinaire ". Pour une espèce au sein de sa chaîne alimentaire, les questions posées se traitent avec des focus variés (avec des outils tour à tour analytiques ou systémiques) et dans des contextes intégrant plus ou moins des contingences culturelles et éthiques. Les actions se définissent alors dans un positionnement selon deux axes: réductionnisme/holisme et objectivité/subjectivité. Notre approche globale est le passage d'un point de vue à l'autre en établissant le lien entre eux. Cette approche globale s'appuie sur une connaissance généraliste associée à une compétence de résolution de problèmes concrets qui sont la spécificité des ingénieurs. Cette démarche, portée dans le cadre de la recherche scientifique et connectée à des réalités culturelles, sociales, économiques, réglementaires, politiques et éthiques, est notre proposition pour participer au développement d'une agriculture biologique soutenable.

\section{Conclusion}

L'enjeu véritable d'une recherche pour la bio n'est-il pas de faire reconnaître la cohérence de ses hypothèses posées tour à tour dans tous les continents, basée sur la maximisation des processus vitaux dans le respect de la nature et des hommes ? Elle représente une rupture idéologique profonde, il ne s'agit pas de comprendre pour maîtriser, mais de questionner de façon continue les acquis pour apprendre tous les jours un peu plus dans l'accompagnement du processus. Alors, I'homme, qu'il soit chercheur ou paysan, législateur ou boulanger, pénètrera progressivement davantage dans la réalité du monde qui le nourrit plutôt que de s'en extraire pour prétendre à une vérité scientifique neutre dont les limites reculent avec la précision de ses outils. Pourrons-nous découvrir toutes les facettes d'une expression de Howard: "Vivre et laisser vivre " ? II invite à la coopération, à la complémentarité, à la synergie. Les multiples définitions de l'agriculture biologique et les pratiques associées sont des déclinaisons culturelles du même principe de coopération avec la vie. Elles sont d'autant plus enrichissantes que chacune apporte un éclairage complémentaire à l'autre.

Aujourd'hui, en Europe, au passage entre deux millénaires, des paysans audacieux ont invité les plantes cultivées à co-évoluer de nouveau avec les pratiques agricoles et le terroir. Dans un monde d'instabilité à tous niveaux, le rôle de l'ingénieur est d'accompagner les praticiens inventant l'agriculture paysanne de demain dans l'observation, la compréhension et la mise en perspective de leurs résultats et de développer un réseau, toujours plus dense, de collecte de données à partager et à mettre en relation entre elles. La démarche de recherche doit couvrir le système alimentaire, stimuler sa diversité, l'aborder du sol au produit pour étudier les interactions des plantes avec leur environnement, de leur produit avec les pratiques culinaires ou de transformation car l'ancrage au terroir est une base indispensable pour la bio.

Cette démarche globale, loin de refuser une démarche disciplinaire, en repousse les limites et les enrichit, en confrontant chacune d'elles aux questions des autres. Le processus itératif de la recherche invite à approfondir et à compléter le panel de disciplines impliquées au fur et à mesure que les questions se posent dans les champs, les fournils ou les assiettes, ou encore dans le bureau du législateur. La construction des connections entre savoirs et savoir-faire créent une transdisciplinarité dynamique 
grâce à I'organisation multi-acteurs et participative, sans se priver d'allers-retours avec la recherche académique pour des approfondissements scientifiques " classiques".

Au cours de ce processus, la création de connaissances n'apparaît alors plus comme un but en soi mais comme un coproduit d'un processus productif et culturel, une nécessité pour avancer ensemble vers un écosystème respecté par tous où "le beau " finira par retrouver une place. On ne parle plus de processus top-down ou bottom-up mais plutôt circulaires. Certains paysans ayant risqué I'aventure participative avec la recherche institutionnelle depuis plusieurs années, se reconnaissent avec un certain plaisir "paysans-chercheurs"; les jeunes doctorants ou ingénieurs rêvent aussi à leur tour d'une terre d'accueil pour devenir (certains le sont déjà) " chercheurs-paysans ". L'organisation de la recherche pour la bio paysanne est un nouveau chantier à ouvrir.

\section{Glossaire - Définitions}

\section{Agriculture biologique ; Organic agriculture}

L'agriculture biologique s'appuie sur l'hypothèse que la production agricole doit reposer sur une coopération avec l'écosystème dans sa globalité en respectant les lois de la nature. Elle optimise par ses pratiques les capacités productrices d'un terroir tout en préservant la santé de diverses communautés au sein de l'écosystème agricole, y compris les organismes du sol, les plantes, les animaux d'élevage et la population. Elle favorise la biodiversité, la fertilité naturelle des sols, l'autonomie des paysans et une alimentation saine à portée de tous. Des principes ont été posés par différents pionniers dans le monde dès le début du XX siècle; elle prend plusieurs formes et désignations selon les cultures et la géographie des lieux.

\section{Agriculture paysanne ; Peasant agriculture}

Elle est définie au cours des années 1990 par des paysans de la Confédération paysanne et de la FADEAR (Fédération associative de développement de l'emploi agricole et rural) : " L'agriculture paysanne doit permettre à un maximum de paysans répartis sur tout le territoire de vivre décemment de leur métier en produisant sur des exploitations à taille humaine une alimentation saine et de qualité, sans remettre en cause les ressources naturelles de demain. Elle doit participer avec les citoyens à rendre le milieu rural vivant dans un cadre de vie apprécié par tous " http://www.confederationpaysannepdl.fr/Qu-est-ce-que--l-agriculture-paysanne.

L'objectif de l'agriculture paysanne est de repenser la place de I'agriculture dans la société en redonnant aux actifs agricoles leur rôle social, économique et écologique afin de considérer la production agricole dans sa globalité, productrice de biens marchands et non marchands dans l'intérêt de la société. Elle est basée sur des principes politiques qui doivent orienter les décisions politiques et de terrain, http://www.agriculturepaysanne.org.

\section{Système alimentaire ; Food system}

Un système alimentaire est l'ensemble des activités de production, d'échange, de transformation, de distribution et de consommation de denrées alimentaires.

\section{Approche multi-acteurs ; Multi-actor approach}

Nous proposons de retenir celle définie par la Commission européenne pour les projets H2020: "L'approche multi-acteurs vise à promouvoir l'innovation basée sur la demande par une participation véritable et soutenue des différents acteurs (utilisateurs finaux tels que les groupes d'agriculteurs/paysans, pêcheurs/groupes de pêcheurs, des conseillers, des entreprises, etc.) tout au long du projet: de la participation à la planification des travaux et à la réalisation d'expériences, pendant leur exécution jusqu'à la diffusion des résultats et jusqu'à la phase de démonstration. L'approche multi-acteurs repose sur le choix adéquat des acteurs-clés avec des formes de savoirs complémentaires (scientifique et pratique)".

\section{Transdisciplinarité ; Transdisciplinarity}

De façon littérale, la transdisciplinarité peut être définie comme une science qui va au-delà et fait le lien entre toutes les disciplines. Elle est de plus en plus conçue comme une coproduction de connaissances par un processus de collaboration qui implique de multiples disciplines scientifiques et des intervenants de secteurs variés représentant l'ensemble de la société. De nombreux scientifiques et philosophes considèrent la transdisciplinarité comme une nécessité en raison de l'évolution des contenus de la connaissance et des grands enjeux contemporains, comme le changement climatique et la perte de la biodiversité. Le défi n'est plus d'accumuler des savoirs fragmentés dans des disciplines bien délimitées, mais d'utiliser les connaissances les plus diverses d'acteurs scientifiques et praticiens pour construire un savoir répondant aux problématiques complexes d'aujourd'hui. 


\section{Recherche multi-acteurs et transdisciplinaire pour des systèmes alimentaires bio et locaux}

par Véronique CHABLE

Ingénieur de recherche à I'INRA (Institut national de la recherche agronomique) Unité de recherche INRA SAD paysage, équipe Biodiversité cultivée et recherche participative INRA-ITAB, Rennes, France

et Estelle SERPOLAY

Chargée de mission à I'ITAB (Institut technique de l'agriculture biologique) Unité de recherche INRA SAD paysage, équipe Biodiversité cultivée et recherche participative INRA-ITAB, Rennes, France

\section{Sources bibliographiques}

[1] OELHAF (R.C.). - Organic agriculture, economic and ecological comparisons with conventional methods. John Wiley \& Sons, New York (1978).

[2] HOWARD (A.). - Testament agricole (1943).

[3] CAPLAT (J.). - L'agriculture biologique pour nourrir l'humanité. Démonstration. Domaine du Possible, Actes sud (2012).

[4] STASSART (P.M.) et JAMAR (D.). - Agriculture biologique et verrouillage des systemes de connaissances. Conventionalisation des filières agroalimentaire bio. Innovations Agronomiques, 4, p. 313-328 (2009) http://orgprints.org/15497/1/43-Stassart.pdf

[5] Agriculture Course : Preface - Rudolf Steiner, Archive

http://wn.rsarchive.org/Lectures/GA327/ English/BDA1958/Ag1958_preface.html\# sthash.ytJdbMAi.dpuf (traduction 1958)

[6] HECKMAN (J.). - A history of organic farming: Transitions from Sir Albert Howard's War in the Soil to the USDA National Organic Program PhD (21 juillet 2007) http://www.westonaprice.org/A-History-ofOrganic-Farming-Transitions-from-SirAlbert-Howard-s-War-in-the-Soil-to-theUSDA-National-Organic-Program.html

[7] CALAME (M.). - Une agriculture pour le XXI siècle: manifeste pour une agronomie biologique. Éditions Charles Léopold Mayer (2007).

[8] DORÉ (T.), MAKOWSKI (D.), MALÉZIEUX (E.), MUNIER-JOLAIN (N.), TCHAMITCHIAN (M.) et TITTONELL (P.). - - Facing up to the paradigm of ecological intensification in agronomy: revisiting methods, concepts and knowledge. Europ. J. Agronomy, 34, p. 197-210 (2011).
[9] BOUCHER (J.). - Précis scientifique et pratique de culture biologique. $4^{\mathrm{e}}$ Édition, "Agriculture et vie ", Angers (1968).

[10] DE SCHUTTER (O.). - Notre modèle agricole mondial est à bout de souffle. Le Monde.fr I 29.04.2014 à $11 \mathrm{~h} 44 \bullet$ Mis à jour le 29.04.2014 à $15 \mathrm{~h} 52$ | Propos recueillis par Gilles van Kote.

[11] WEZEL (A.), BELLON (S.), DORE (T.), FRANCIS (C.), VALLOD (D.) et DAVID (C.). - Agroecology as a science, a movement and a practice. A review. Agronomy for Sustainable Development, 29, p. 503-515, DOI:10.1051/agro/2009004 (2009).

[12] ALTIERI (M.A.). - Agroecology: the science of sustainable agriculture. Westview Press (1995).

[13] WEZEL (A.) et SOLDAT (V.). - A quantitative and qualitative historical analysis of the scientific discipline of agroecology. International Journal of Agricultural Sustainability, 7, p. 3-18, DOI:10.3763/ijas.2009.0400 (2009).

[14] DAVID (C.), WEZEL (A.), BELLON (S.), DORÉ (T.) et MALÉZIEUX (E.). - Agroécologie (2011)

http://mots-agronomie.inra.fr/ mots-agronomie.fr/index.php/Agroécologie

[15] LE FOLL (S.). - Projet agroecologique pour la France (2012) http://agriculture.gouv.fr/IMG/pdf/projetagroecologique-2013_cle43b56c.pdf

[16] LIEBLEIN (G.), CAPORALI (F.), VON FRAGSTEIN (P.) et FRANCIS (C.). - Research - Teaching integration in agroecology and organic farming. (2008) http://orgprints.org/1 1979/1/11979.pdf

[17] ALTIERI (M.A.). - Agroecology, small farms, and food sovereignty. Monthly Review, 61(3), vol. 61, issue 3, juil.-août 2009 http://monthlyreview.org/2009/07/01/ agroecology-small-farms-and-foodsovereignty/

[18] http://www.hort.purdue.edu/newcrop/ hort_306/reading/Reading\%2031-2.pdf

[19] BELLON (S.), GAUTRONNEAU (Y.), RIBA (G.), SAVINI (I.) et SYLVANDER (B.). - L'agriculture biologique et I'INRA, vers un programme de recherche. Livre blanc (2000).

[20] GATE (P.). - Les nouvelles techniques agricoles - évolutions; L'agro-écologie carrefour entre l'agronomie et l'écologie. Conférence par Philippe GATE Directeur Scientifique de ARVALIS Institut du végétal (2014) http://www.interactif-agriculture.org/pages/ conferences/2014-I-agro-ecologie.php

[21] http://presse.inra.fr/Ressources/ Communiques-de-presse/Agro-ecologie

[22] LOUBENS (A.). - Le rapport de I'INRA sur l'agriculture biologique fait polémique. Actualités, 6 mars 2014

http://institut.inra.fr/Missions/Eclairer-lesdecisions/Etudes/Toutes-les-actualites/ Rapport-Agricultures-hautes-performances, et sa controverse

http://www.techniques-ingenieur.fr/actualite/ biotech-chimie-thematique_6343/le-rapportde-I-inra-sur-I-agriculture-biologique-faitpolemique-article_90361/

[23] SOLIBAM. - Strategies for organic and low input integrated breeding and management. FP7 (2010-2014)

http://www.solibam.eu/modules/addresses/ viewcat.php?cid $=1$

[24] L'agroécologie? C'est étudier l'agriculture avec les regards croisés de l'agronome et de l'écologue.

http://www.inra.fr/Grand-public/Agriculturedurable/Tous-les-dossiers/L-agroecologievous-connaissez 
[25] JOUVE (P.). - Périodes et ruptures dans l'évolution des savoirs agronomiques et de leur enseignement. In: Histoire et agronomie. Entre ruptures et durée, Éd. IRD Collection Colloques et Séminaires (2007).

[26] CHEVASSUS AU LOUIS (B.). - Refonder la recherche agronomique: leçon du passé, enjeu du siècle. DR de recherche INRA, Leçon inaugurale du groupe ESA, Angers, sept. 2006

[27] CRESSON (C.). - L'organisation institutionnelle de l'agriculture Bio. Dossier agriculture biologique. AgroMag, oct.-nov.-déc. 2010.

[28] http://www.organic-research.net/2433.html?\&L=jsffvjbug

[29] GOETHE. - La métamorphose des plantes. Éd. Triades, Paris, $3^{\mathrm{e}}$ édition françaises (1999).

[30] http://www.encyclopedie-dd.org/ encyclopedie/terre/5-2-les-differentssecteurs/l-agriculture-biologique.html

[31] BAARS (T.). - Experiential science; towards an integration of implicit and reflected prac titioner expert knowledge in the scientific development of organic farming. Journal Agric Environ Ethics, 24(6), p. 601-628, DOI 10.1007/s10806-010-9281-3 (2011).

[32] LIEBLEIN (G.), ØSTERGAARD (E.) et FRANCIS (C.). - Becoming an agroecologist through action education. Int. J. Agric. Sustainability, 2(3), p. 147-153 (2005).

[33] ERNESTO MÉNDEZ (V.), BACON (C.M.) et COHEN (R.). - Agroecology as a transdisciplinary, participatory, and action-oriented approach. Agroecology and Sustainable Food Systems, 37, p. 3-18 (2013).

[34] PAUL (J.). - The farm as organism : the foun dational idea of organic agriculture elementals. Journal of Bio-Dynamics Tasmania, 83, p. 14-18 (2006) http://orgprints.org/10138/1/10138.pdf

[35] BAARS (T.). - Reconciling scientific approaches for organic farming research. Wageningen NL: Dissertation Wageningen University, 346 p. (2002).

[36] RAMMEL (C.) et VAN DEN BERGH (J.C.J.M.). - Evolutionary policies for sustainable development: adaptive flexibility and risk minimizing. Ecological Economics, 47, p. 121-133 (2003).

[37] ftp://ftp.fao.org/docrep/fao/010/i0112f/i0112f09.pdf

[38] Définition de la variété figurant à l'article 1.IV de I'acte de 1991 de la convention de I'UPOV http://www.upov.int/edocs/expndocs/fr/ upov exn var 1.pdf

[39] http://www.gnis-pedagogie.org/lexique.html

[40] MURPHY (K.), CARTER (A.H.) et JONES (S.S.). - Evolutionary breeding and climate change. In: Genomics and Breeding for Climate-Resilient Crops, Concepts and Strategies, KOLE (C.) (ed.), Springer, vol. 1, p. 377-389 (2013).

[41] CECCARELLI (S.), GALIE (A.) et GRANDO (S.). - Participatory breeding for climate change-related traits. Genomics and Breeding for Climate-Resilient Crops, Concepts and Strategies, KOLE (C.) (ed.), Springer, vol. 1, p. 331-376 (2013)

[42] CECCARELLI (S.), GRANDO (S.), BAUM (M.) et UDUPA (S.M.). - Breeding for drought resistance in a changing climate. In Challenges and Strategies of Dryland Agriculture, RAO (S.C.) et RYAN (J.) (ed.), p. 167-190 (2004).

[43] ZHU (Y.Y.), CHEN (H.), FAN (J.), WANG (Y.), $\mathrm{LI}(\mathrm{Y}$.$) , CHEN (J.) et FAN (J.). - Genetic diver-$ sity and disease control in rice. Nature, 406, p. $718-722(2000)$.
[44] DE VALLAVIEILLE-POPE (C.). - Management of disease resistance diversity of cultivars of a species in single fields: controlling epidemics. C. R. Biol., 327, p. 611-620 (2004).

[45] OSTERGAARD (H.), FINCKH (M.R.), FONTAINE (L.), GOLDRINGER (I.), HOAD (S.P.) KRISTENSEN (K.), LAMMERS VAN BUEREN (E.T.), MASCHER (F.), MUNK (L.) et WOLFE (M.S.). - Time for a shift in crop production : embracing complexity through diversity at all levels. J. Sci. Food Agric., 89, p. 1439-1445 (2009).

[46] MULUMBA (JW), NANKYA (R), ADOKORACH (J.), KIWUKA (C.), FADDA (C.), DE SANTIS (P.) et JARVIS (D.I.). - A risk-minimizing argument for traditional crop varietal diversity use to reduce pest and disease damage in agricultural ecosystems of Uganda. Agric. Ecosyst. Environ, 157, p. 70-86 (2012).

[47] WIMP (G.M.), YOUNG (W.P.), WOOLBRIGHT (S.A.), MARTINSEN (G.D.), KEIM (P.) et WHITHAM (T.G.). - Conserving plant genetic diversity for dependant animal communities. Ecol. Lett., 7, p. 776-780 (2004).

[48] BAILEY (J.K.), SCHWEITZER (J.A.), UBEDA (F.), KORICHEVA (J.), Le ROY (C.J.), MADRITCH (M.D.), REHILL (B.J.), BANGERT (R.K.), FISCHER (D.G.), ALLAN (G.J.) et WHITHAM (T.G.). - From genes to ecosystems : a synthesis of the effects of plant genetic factors across levels of organization. Phil. Trans. R. Soc., 364, p. 1607-1616 (2009).

[49] CHATEIL (C.), GOLDRINGER (I.), TARALLO (L.), KERBIRIOU (C.), LE VIOL (I.), PONGE (J.F.), SALMON (S.), GACHET (S.) et PORCHER (E.). - Crop genetic diversity benefits farmland biodiversity in cultivated fields. Agric. Ecosyst. Environ., 171, p. 25-32 (2013).

[50] FINCKH (M.), GACEK (E.), GOYEAU (H.), LANNOU (C.) et MERZ (U.) et al. - Cereal variety and species mixtures in practice, with emphasis on disease resistance. Agronomie, EDP Sciences, 20(7), p. 813-837 (2000).

[51] MILLE (B.), FRAJ (M.B.), MONOD (H.) et DE VALLAVIEILLE-POPE (C.). - Assessing four-way mixtures of winter wheat cultivars from the performances of their two-way and individual components. European J. Plant. Pathology, 114, p. 163-173 (2006).

[52] WOLFE (M.S.). - Crop strength through diversity. Nature, 406, p. 681-682 (2000).

[53] WOLFE (M.S.), BARESEL (J.P.), DESCLAUX (D.), GOLDRINGER (I.), HOAD (S.), KOVACS (G.), LÖSCHENBERGER (F.), MIEDANER (T.), ØSTERGÅRD (H.) et LAMMERTS VAN BUEREN (E.T.). - Developments in breeding cereals for organic agriculture in Europe. Euphytica, 163, p. 323-346 (2008).

[54] GOLDRINGER (I.), PROUIN (C.), ROUSSET (M.), GALIC (N.) et BONNIN (I.). - Rapid differentiation of experimental populations of wheat for heading-time in response to local climatic conditions. Annals of Botany, 98, p. 805-817 (2006).

[55] RHONÉ (B.), REMOUE (C.), GALIC (N.), GOLDRINGER (I.) et BONNIN (I.). - Insight into the genetic bases of climatic adaptation in experimentally evolving wheat populations. Molecular Ecology, 17, p. 930-943 (2008).

[56] 10 concepts clés de SOLIBAM - Cultivons la diversité http://www.solibam.eu/

[57] MA,2005. - Ecosystems and human well-being: synthesis. Millennium Ecosystem Assessment, Island Press, Washington DC (2005)

http://www.millenniumasses.0sment.org/ documents/document.356.aspx.pdf
[58] DORÉ (C.) et VAROQUAUX (F.). - Histoire et amélioration de 50 plantes cultivées. Éditions INRA (2006).

[59] GALLAIS (A.). - Hétérosis et variétés hybrides en amélioration des plantes. Editions Quae INRA Versailles (2009).

[60] GOFFAUX (R.), GOLDRINGER (I.), BONNEUIL (C.), MONTALENT (P.) et BONNIN (I.), - Quels indicateurs pour suivre la diversité génétique des plantes cultivées? Le cas du blé tendre cultivé en France depuis un siècle. Rapport FRB, Série Expertise et synthèse, $44 \mathrm{p}$. (2011).

[61] GOLDRINGER (I.) et al. - From landraces to mixtures and to composite cross popu lations: a range of initial populations to star evolutionary participatory plant breeding International Symposium on Evolutionary Breeding in Cereals, Aston University, Birmingham, UK, 21 janv. 2013.

[62] BONNEUIL (C.) et THOMAS (F.). - Gènes, pouvoirs et profits. Quae-FPH (2009).

[63] BONNEUIL (C.) et DEMEULENAERE (E.). Vers une génétique de pair à pair. L'émergence de la sélection participative. Ouvrage Des sciences citoyennes, Éds CHARVOLIN, MICOUD (A.), ELLIS (R.), Paris, Éd. de I'Aube (2007).

[64] CHABLE (V.), LOUWAARS (N.), HUBBARD (K.), BAKER (B.) et BOCCI (R.). - Plant breeding, variety release and seed commercialisation: laws and policies of concern to the organic sector. LAMMERTS VAN BUEREN (E.T.) et MYERS (J.R.) (Eds), Organic Crop Breeding. Wiley-Blackwell, Hoboken, NY, États-Unis, ISBN: 978-0-470-95858-2 (2012).

[65] GOUYON (P.H.). - Conférence à l'école normale supérieure OHNK, juin 2014 http://www.les-ernest.fr/la-biodiversite/

[66] HERVIEU (B.). - L'amélioration des plantes, un domaine emblématique pour I'INRA. histoire, identité, horizons. Actes du colloque "L'Amélioration des Plantes, continuités et ruptures », BOISTARD (P.), SABBAGH (C.) ET SAVINI (I.), éditeurs, Montpellier, 17-18 oct. 2002 (2004).

[67] VAN DER PLOERG (J.D.). - The new peasantries - struggles for autonomy and sustainability in an era of empire and globalization. Earthscan (London) (2008) http://www.jandouwevanderploeg.com/ EN/the-new-peasantries/

[68] http://www.natureetprogres.org/ nature_et_progres/natureetprogres.html

[69] http://www.itab.asso.fr/downloads/ Fiches techniques_semences/ fiche $\% 20$ semencereglementationsmini.pdf

[70] DELIVERABLE D1.4. - Report on the diversity of low-input and organic systems, the stakeholders' expectations and innovations concerning crop management and breeding for low-input/organic (2013) http://www.solibam.eu/modules/ wfdownloads/viewcat.php?cid=7

[71] CHABLE (V.), CONSEIL (M.), SERPOLAY (E.) et LE LAGADEC (F.). - Organic varieties for cauliflowers and cabbages in Brittany: from genetic resources to participatory plant breeding. Euphytica, 164, p. 521-529 (2008) http://dx.doi.org/10.1007/s10681-008-9749-7

[72] CHABLE (V.), DAWSON (J.), BOCCl (R.) et GOLDRINGER (I.). - Seeds for organic agriculture: development of participatory plant breeding and farmers' networks in France. In: Organic farming, prototype for sustainable agricultures, BELLON (S.) et PENVERN (S.) (Eds), Springer, Berlin (2014). 
[73] VINDRAS-FOUILLET (C.) et CHABLE (V.). - Integrative breeding strategies to improve sensory qualities of wheat bread. In CHABLE (V.), GOLDRINGER (I.), HOWLETT (S.A.), BARBERI (P.), MIKO (P.), MENDES- MOREIRA (P.M.R.), RAKSZEGI (M.), ØSTERGÅRD (H.), BORGEN (A.), FINCKH (M.R.), PEDERSEN (T.), BOCCI (R.) (Eds.), Diversity strategies for organic and low input agricultures and their food systems, Book of abstracts of SOLIBAM final congress, $7^{\text {th }}-9^{\text {th }}$ July 2014, Nantes, France, p. 80-81 (2014).

[74] VINDRAS (C.). - Évaluation de la qualité sensorielle de produits pour la sélection participative en agriculture biologique: cas du blé et du brocoli. Thèse de I'Université Européenne de Bretagne - École Doctorale : "VIE AGRO SANTÉ» (2014).

[75] BOCCI (R.) et CHABLE (V.). - Semences paysannes en Europe : enjeux et perspectives. Cahiers d'études et de recherche francophones/Agricultures, 17(2), p. 216-221 (2008).
[76] Le Gnis et I'UFS souhaitent que les échanges reprennent rapidement Publié le jeudi 13 mars 2014 - 17 h 29 (2014)

http://www.lafranceagricole.fr/actualite-

agricole/reglement-sur-les-semences-ue-

le-gnis-et-l-ufs-souhaitent-que-les-echanges-reprennent-rapidement-85582.

html\#BmOIVCOWbFOaGeLI.99

[77] ReSoRIV. - Reconnaissance sociale et réglementaire de l'innovation variétale par la sélection participative pour les agricultures biologique et paysanne (2012-2013)

http://www.programme-repere.fr/

wp contesnt/uploads/Fiche_ReSoRIV.pdf

\section{Sites Internet}

Projets marquants

http://www.sad.inra.fr/Toutes-les-actualites/Farm-Seed-Opportunities https://www.solibam.eu ; https://colloque6.inra.fr/solibam2014

https://www.diversifood.eu http://www.cobra-div.eu/

http://sciencescitoyennes.org/rubrique/nos-propositions-et-actions/nos act ivites/projet-reconnaissance-sociale-et-reglementaire-de-linnovation-varietale-resoriv-repere-2/

\section{Annuaire}

\section{En France}

http://moulon.inra.fr/index.php/equipes/deap http://www.itab.asso.fr/

http://www.semencespaysannes.org/

http://www.agrobioperigord.fr/produire-bio/biodiversite-cultivee

http://kaolkozh5.blogspot.fr/

https://www6.versailles-grignon.inra.fr/sadapt/Equipe-Proximites

http://www6.toulouse.inra.fr/agir/

https://www6.montpellier.inra.fr/diascope/

En Europe

http://www.efrc.com/

http://www fibl.org/

http://www.louisbolk.org/

http://www.eco-pb.org/

http://www.agroscope.ch/

http://www.dtu.dk/

http://portal.ipc.pt/portal http://www.itqb.unl.pt/ http://www.ias.csic.es/ http://www.sssup.it/

http://www.wzw.tum.de/

http://www.mgki.hu/

http://www.unipi.it/

http://www.unibo.it/

http://www.moa.gov.cy/

https://www.luke.fi/

http://omki.org/

http://liberatediversity.org/

http://www.semirurali.net/

http://www redsemillas info/

https://www arche-noas.inf/

https://www. prospecierara.ch/

https://www.prospecier 
GAGNEZ DU TEMPS ET SÉCURISEZ VOS PROJETS

EN UTILISANT UNE SOURCE ACTUALISÉE ET FIABLE

Techniques de l'Ingénieur propose la plus importante collection documentaire technique et scientifique en français !

Grâce à vos droits d'accès, retrouvez l'ensemble des articles et fiches pratiques de votre offre, leurs compléments et mises à jour, et bénéficiez des services inclus.

\begin{tabular}{|c|c|c|c|}
\hline $\begin{array}{c}\text { RÉDIGÉE ET VALIDÉE } \\
\text { PAR DES EXPERTS }\end{array}$ & $\begin{array}{c}\text { MISE À JOUR } \\
\text { PERMANENTE }\end{array}$ \\
SUR TOUS SUPPORTS \\
NUMÉRIQUES
\end{tabular}

\title{
$>+$ de 350000 utilisateurs \\ $>+$ de 10000 articles de référence \\ $>+$ de 80 offres
}

\section{$>15$ domaines d'expertise}

\author{
Automatique - Robotique \\ Biomédical - Pharma \\ Construction et travaux publics \\ $\bigcirc$ Électronique - Photonique \\ $\bigcirc$ Énergies \\ Environnement - Sécurité \\ Génie industriel \\ Ingénierie des transports
}

Innovation

Matériaux

Mécanique

Mesures - Analyses

Procédés chimie - Bio - Agro

Sciences fondamentales

Technologies de l'information

Pour des offres toujours plus adaptées à votre métier, découvrez les offres dédiées à votre secteur d'activité

Depuis plus de 70 ans, Techniques de l'Ingénieur est la source d'informations de référence des bureaux d'études, de la R\&D et de l'innovation. 


\section{LES AVANTAGES ET SERVICES}

\section{compris dans les offres Techniques de l'Ingénieur}

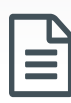

Accès illimité aux articles en HTML

Enrichis et mis à jour pendant toute la durée de la souscription

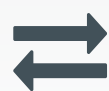

Questions aux experts*

Les meilleurs experts techniques et scientifiques vous répondent

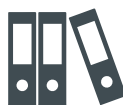

Archives

Technologies anciennes et versions antérieures des articles

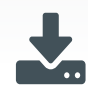

Téléchargement des articles au format PDF

Pour un usage en toute liberté

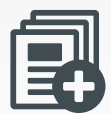

Articles Découverte

La possibilité de consulter des articles en dehors de votre offre

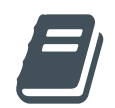

Impression à la demande

Commandez les éditions papier de vos ressources documentaires
Consultation sur tous

les supports numériques

Des contenus optimisés

pour ordinateurs, tablettes et mobiles
Dictionnaire technique multilingue 45000 termes en français, anglais, espagnol et allemand

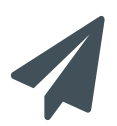

Alertes actualisations Recevez par email toutes les nouveautés de vos ressources documentaires

\section{ILS NOUS FONT CONFIANCE}

AgroParisTech

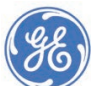
Schneider
(9) AIRBUS

L'OREAL Leroy-Somer

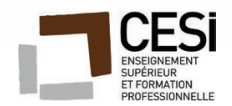

ecnes

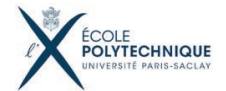
S SAFRAN

veolia Sagemсом

SyASSAULT

eNGie SAINT-GOBAIN

\section{www.techniques-ingenieur.fr}

